TRANSACTIONS OF THE

AMERICAN MATHEMATICAL SOCIETY

Volume 351, Number 6, Pages 2515-2538

S 0002-9947(99)02221-7

Article electronically published on February 15, 1999

\title{
THE IDEAL STRUCTURE OF SOME ANALYTIC CROSSED PRODUCTS
}

\author{
MIRON SHPIGEL
}

\begin{abstract}
We study the ideal structure of a class of some analytic crossed products. For an $r$-discrete, principal, minimal groupoid $G$, we consider the analytic crossed product $C^{*}(G, \sigma) \times_{\alpha} \mathbb{Z}_{+}$, where $\alpha$ is given by a cocycle $c$.

We show that the maximal ideal space $\mathcal{M}$ of $C^{*}(G, \sigma) \times_{\alpha} \mathbb{Z}_{+}$depends on the asymptotic range of $c, R_{\infty}(c)$; that is, $\mathcal{M}$ is homeomorphic to $\overline{\mathbb{D}} \mid R_{\infty}(c)$ for $R_{\infty}(c)$ finite, and $\mathcal{M}$ consists of the unique maximal ideal for $R_{\infty}(c)=\mathbb{T}$. We also prove that $C^{*}(G, \sigma) \times_{\alpha} \mathbb{Z}_{+}$is semisimple in both cases, and that $R_{\infty}(c)$ is invariant under isometric isomorphism.
\end{abstract}

\section{INTRODUCTION}

Our aim is to study the ideal structure of a certain class of analytic crossed products (or semicrossed products according to J. Peters [P1]) $A \times_{\alpha} \mathbb{Z}_{+}$.

Peters [P1] defined the concept of a semicrossed product in a more general way for an endomorphism $\alpha$. He also studied the case when $\alpha$ is an automorphism and $A$ is a commutative $C^{*}$-algebra. In their joint work De Alba and Peters [DP] classified semicrossed products of finite-dimensional $C^{*}$-algebras. Also we must mention the work of McAsey and Muhly [MM], concerning representations of non-self-adjoint crossed products.

Another paper by Peters [P2], devoted to inductive limits of matrix algebras of holomorphic functions, is closely related to our situation. In that paper Peters proved that such an inductive limit (with connecting homomorphism of a special form) is isomorphic to an analytic crossed product $U \times_{\alpha} \mathbb{Z}_{+}$, where $U$ is a UHF algebra and $\alpha$ is a certain automorphism of $U$.

The main questions in our investigation concern the maximal ideal structure and semisimplicity of our class of analytic crossed products.

In this paper we study analytic crossed products $A \times{ }_{\alpha} \mathbb{Z}_{+}$where $A$ is a groupoid $C^{*}$-algebra. The groupoid will be assumed to be locally compact, $r$-discrete, principal, minimal and amenable (for definitions, see [R], or section 2 of this paper). Such a groupoid $G$ can be viewed as an equivalence relation on a certain locally compact, second countable, Hausdorff space $X$. Given a 2-cocycle $\sigma$ on $G$, we write $C^{*}(G, \sigma)$ for the algebra introduced by Renault in [R] (see also [MS], [S]) and consider the analytic crossed product $C^{*}(G, \sigma) \times_{\alpha} \mathbb{Z}_{+}$. for an automorphism $\alpha$ which preserves elementwise the diagonal algebra $C^{*}\left(G^{(0)}, \sigma\right) \subseteq C^{*}(G, \sigma)$. It is known that every such automorphism is given by a 1-cocycle $c: G \rightarrow \mathbb{T}$ (see $[\mathrm{K}]$, Corollary 4 ). The $C^{*}$-crossed product $C^{*}(G, \sigma) \times_{\alpha} \mathbb{Z}$ is isomorphic to the $C^{*}$-algebra of another (so

Received by the editors December 2, 1996.

1991 Mathematics Subject Classification. Primary 47D25; Secondary 46H10, 46L05.

(C)1999 American Mathematical Society 
called skew-product) groupoid, $G(c)$ [R, Theorem 5.7, p.118]. The analytic crossed product $C^{*}(G, \sigma) \times_{\alpha} \mathbb{Z}_{+}$is a subalgebra of this $C^{*}$-algebra. In this paper we study analytic crossed products, using the groupoid approach, which was developed by $\mathrm{P}$. Muhly and B. Solel ([MS], [S]).

In section 2 we prove that the description of the maximal ideal space of $C^{*}(G, \sigma)$ $\times_{\alpha} \mathbb{Z}_{+}$depends on the asymptotic (or essential) range $R_{\infty}(c)$ of the cocycle $c$, which coincides with the Connes spectrum of $\alpha, \Gamma(\alpha)([\mathrm{R}])$.

Since $R_{\infty}(c)$ is a closed subgroup of $\mathbb{T}$, it is either $\mathbb{T}$ or a finite subgroup. We prove (see Corollary 2.12) that, if $R_{\infty}(c)=\mathbb{T}$, then there exists a unique maximal ideal in $C^{*}(G, \sigma) \times_{\alpha} \mathbb{Z}_{+}$and, moreover, the set of all proper, two-sided, closed ideals forms a countable decreasing set in this case.

If $R_{\infty}(c)$ is a finite subgroup $\mathbb{T}$, then there is a one-to-one correspondence between the closed disc $\overline{\mathbb{D}}=\{z \in \mathbb{C}, \quad|z| \leq 1\}$ and the maximal ideal space $\mathcal{M}$ of $C^{*}(G, \sigma) \times_{\alpha} \mathbb{Z}_{+}$(see Theorem 2.15). Moreover, we prove in section 4 that $\mathcal{M}$ with the hull-kernel topology is homeomorphic to $\overline{\mathbb{D}} / R_{\infty}(c)$, where $\overline{\mathbb{D}}$ is provided with the hull-kernel topology of $A(\mathbb{D})$ (Theorem 4.2).

In section 3 we show that in both cases we obtain a semisimple algebra.

In addition, in section 5, we prove (Theorem 5.1) that the asymptotic range $R_{\infty}(c)$ is invariant with respect to isometric isomorphism of the analytic crossed products, i.e. if $A=C^{*}(G, \sigma)$ and $A \times_{\alpha_{1}} \mathbb{Z}_{+}$is isometrically isomorphic to $A \times_{\alpha_{2}} \mathbb{Z}_{+}$ (for $\alpha_{i}, i=1,2$, as above), then $\Gamma\left(\alpha_{1}\right)=R_{\infty}\left(c_{1}\right)=R_{\infty}\left(c_{2}\right)=\Gamma\left(\alpha_{2}\right)$.

Note that every simple AF algebra (in particular, UHF-algebra) is a $C^{*}$-algebra of a groupoid of the kind we study $[\mathrm{R}]$.

Thus we generalize the results of [P2], concerning maximal ideal space for the finite Connes spectrum, and we obtain an affirmative answer to his conjecture concerning the case $\Gamma(\alpha)=\mathbb{T}$ (see [P2, main theorem of IV.13 and Remark IV.14]).

We believe that our results concerning the structure of the maximal ideal space of the analytic crossed products and its relation to the Connes spectrum might hold for more general analytic crossed products. However our methods here make use of the underlying groupoid and do not seem to apply in general.

The author is grateful to Professor B. Solel for his advice and help in writing this paper.

\section{Maximal ideals of $C^{*}(G, \sigma) \times_{\alpha} \mathbb{Z}_{+}$}

We intend to study the analytic crossed product $C^{*}(G, \sigma) \times_{\alpha} \mathbb{Z}_{+}$, where $C^{*}(G, \sigma)$ is the $C^{*}$-algebra of a certain $r$-discrete, principal groupoid.

Our first aim is to describe the space of maximal ideals of this algebra. Let us introduce the terminology and notation that we shall use.

Throughout, $X$ will denote a second countable, locally compact Hausdorff space and $G \subseteq X \times X$ will be an equivalence relation on $X$. Then $G$ has a natural groupoid structure as follows. For $x=\left(x_{1}, x_{2}\right), y=\left(y_{1}, y_{2}\right)$, where $x_{i}, y_{i} \in X$, $i=1,2$, and $x_{1} \sim x_{2}$ and $y_{1} \sim y_{2}$ we set

$$
x^{-1}=\left(x_{2}, x_{1}\right)
$$

and also

$$
x y=\left(x_{1}, y_{2}\right)
$$

iff $x_{2}=y_{1}$, and the product is undefined otherwise. 
It is easy to see that, with respect to these relations, $G$ will be a principal groupoid $([\mathrm{R}])$.

Denote by $G^{2}=\left\{\left(x_{1}, y_{1}\right),\left(x_{2}, y_{2}\right) \mid y_{1}=x_{2}\right\}$, and by the set of composable pairs in $G ; G^{(0)}=\{(x, x) ; x \in X\}$ the unit space of $G$.

We can identify $G^{(0)}$ and $X$ using the map $x \rightarrow(x, x)$. We also assume that $G$ is equipped with a topology that satisfies the following properties:

(1) $G$ is a locally compact Hausdorff space,

(2) the maps $x \rightarrow x^{-1}$ from $G$ into $G$ and $(x, y) \rightarrow x y$ from $G^{2}$ into $G$ are continuous, where the topology on $G^{2}$ is induced by the product topology of $G \times G$,

(3) the map $x \rightarrow(x, x)$ from $X$ into $G$ is a homeomorphism onto its range, $G^{0}$, and

(4) $G^{(0)}$ is an open subset of $G$.

Such a groupoid is called an $r$-discrete groupoid.

We assume also that we have a base of compact open $G$-sets for the topology of $G$. Recall that a subset $\tau \subseteq G$ is called a $G$-set if the restictions of $r$ and $s$ to $\tau$ are one-to-one, where for $x=\left(x_{1}, x_{2}\right), r(x)=x_{1}$ and $s(x)=x_{2}$.

Note, in addition, that every equivalence class in $X$ with respect to $G$ is a countable discrete space ([R, Lemma 2.7]).

We assume also that $G$ is amenable (for the definition, see [R], II.3).

Let $\sigma: G^{2} \rightarrow \mathbb{T}$ be a continuous 2-cocycle, i.e. a continuous mapping such that

$$
\sigma(x y, z) \sigma(x, y)=\sigma(y, z) \sigma(x, y z) \text { whenever }(x, y) \text { and }(y, z) \text { lie in } G^{2}
$$

and

$$
\sigma\left(x, x^{-1}\right)=\sigma^{-1}\left(x^{-1}, x\right) .
$$

Now we can construct the $C^{*}$ algebra $C^{*}(G, \sigma)$ as follows: first, define a $*$ algebra structure on $C_{c}(G, \sigma)$ (the continuous functions with compact support on $G$ ) in the following way:

$$
f * g(x, y)=\sum_{z \in[x]} f(x, z) \cdot g(z, y) \cdot \sigma((x, z),(z, y))
$$

and

$$
f^{*}(x, y)=\overline{f(y, x)}
$$

Finally, we write $C^{*}(G, \sigma)$ for the enveloping $C^{*}$-algebra of $C_{c}(G, \sigma)$ (see also [R], [MS]).

Let $c: G \rightarrow \mathbb{T}$ be a continuous cocycle, i.e. a continuous mapping which satisfies the following properties:

$$
\begin{gathered}
c(x, y) \cdot c(y, z)=c(x, z), \\
c(x, y)=\overline{c(y, x)} .
\end{gathered}
$$

Now we define a new groupoid $G(c) . G(c)=G \times \mathbb{T}$ as a set with the following operations:

$$
\begin{gathered}
((x, y), t)^{-1}=((y, x), c(x, y) t), \\
((x, y), t) \cdot((y, z), c(x, y) t)=((x, z), t),
\end{gathered}
$$

and the product is undefined otherwise.

We can see that in this case $G(c)$ is also an $r$-discrete principal groupoid. 
Thus the $C^{*}$ algebra $C^{*}(G(c), \sigma)$ is defined as the enveloping $C^{*}$ algebra for $C_{c}(G(c), \sigma)$, where for every $f, g \in C_{c}(G(c), \sigma)$ we have

$$
\begin{gathered}
f * g((x, y), t)=\sum_{z \in[x]} f((x, z), t) \cdot g((z, y), c(x, z) t) \cdot \sigma((x, z),(z, y)), \\
f^{*}((x, y), t)=\overline{f((y, x), c(x, y) t)} .
\end{gathered}
$$

Every continuous cocycle $c: G \rightarrow \mathbb{T}$ as above gives rise to an automorphism of the $C^{*}$-algebra $C^{*}(G, \sigma)$ by the formula

$$
\alpha(f)(x, y)=c(x, y) \cdot f(x, y) \text { for every } f \in C^{*}(G, \sigma), \quad(x, y) \in G .
$$

It follows from $[\mathrm{R}$, Th. 5.7 , p. 118] that

$$
C^{*}(G, \sigma) \times_{\alpha} \mathbb{Z} \cong C^{*}(G(c), \sigma) .
$$

The crossed product $C^{*}(G, \sigma) \times{ }_{\alpha} \mathbb{Z}$ is generated by the algebra $C^{*}(G, \sigma)$ and a unitary operator $V$. It is spanned by elements of the form $V^{n} \cdot f$ where $f \in C^{*}(G, \sigma)$. The isomorphism carries $V^{n} \cdot f$ into the function $\widehat{V^{n} \cdot f}((x, y), t)=f(x, y) \bar{t}^{\bar{n}}$.

Now we also have $C^{*}(G, \sigma)=C_{\text {red }}^{*}(G, \sigma)$ (because $G$ is amenable; for more details, see $[R])$.

Let $A$ be the analytic crossed product associated with $\alpha$, that is the Banach subalgebra of $C^{*}(G, \sigma) \times{ }_{\alpha} \mathbb{Z}$ which is the closure of $\operatorname{span}\left\{C^{*}(G, \sigma) V^{n}, \quad n \geq 0\right\}$, where $V$ is a generator of the crossed product $C^{*}(G, \sigma) \times{ }_{\alpha} \mathbb{Z}$ (for a general definition, see also $[\mathrm{P} 1])$.

From now on we identify $A$ with its image in $C^{*}(G(c), \sigma)$ with respect to the above-mentioned isomorphism.

Given a function $f \in C^{*}(G, \sigma)$ and a point $(x, y) \in G$, we write $f(x, y)$ for the function $f(x, y)(t)=f((x, y), t)$. Clearly $f(x, y) \in C(\mathbb{T})([\mathrm{R}$, Prop Ii 4.2 (II)].

Lemma 2.1. For every $f \in A$ and every $(x, y) \in G, f(x, y) \in A(\mathbb{D})$.

Proof. Suppose that $f$ as a function in $C^{*}(G, \sigma) \times_{\alpha} \mathbb{Z}$ has the following form:

$$
f=\sum_{n=0}^{\infty} u_{n} V^{n},
$$

where $\sum_{n=0}^{\infty}\left\|u_{n}\right\|<+\infty, u_{n} \in C^{*}(G, \sigma)$. Then by [R, Th. 5.7] the image of $f$, say $\hat{f}$, with respect to above mentioned isomorphism is as follows:

$$
\hat{f}=\sum_{n=0}^{\infty} \alpha^{-n}\left(u_{n}\right) e^{-i n t} .
$$

(i.e. $\left.\hat{f}((x, y), t)=\sum \alpha^{-n}\left(u_{n}\right)(x, y) t^{\bar{n}}\right)$. Since $\left\|\alpha^{-n}\left(u_{n}\right)\right\|=\left\|u_{n}\right\|$ and $\left\|u_{n}\right\|_{\infty} \leq$ $\left\|u_{n}\right\|$ for every $u \in C^{*}(G, \sigma)$ ([R, Prop. 4.2 (II)] ), we have

$$
\sum_{n=0}^{\infty}\left|\alpha^{-n}\left(u_{n}\right)(x, y)\right|<+\infty, \quad(x, y) \in G .
$$

Hence by $(1.2) \hat{f}(x, y) \in A(\mathbb{D})$ for every $(x, y) \in G$ (here we identify $\sum_{n=0}^{\infty} a_{n} e^{-i n t} \in$ $C(\mathbb{T})$ with $\left.\sum_{n=0}^{\infty} a_{n} \cdot z^{n} \in A(\mathbb{D})\right)$. But functions of the kind (1.1) are dense in 
$C^{*}(G, \sigma) \times_{\alpha} \mathbb{Z}$ by definition. Then, if we take now an arbitrary $f \in A=C^{*}(G, \sigma) \times_{\alpha}$ $\mathbb{Z}_{+}$, there is a sequence $\left\{f_{n}\right\}$ satisfying (1.1) such that $f_{n} \rightarrow f$ in norm. Then $\hat{f}_{n} \rightarrow \hat{f}$ in $C^{*}(G(c), \sigma)$. Hence $\hat{f}_{n} \rightarrow \hat{f}$ in the norm $\|\cdot\|_{\infty}$. Now fix an arbitrary $(x, y) \in G$. Then $\hat{f}_{n}(x, y) \rightarrow \hat{f}(x, y)$ in the supremum norm. Therefore, $\hat{f}(x, y) \in$ $A(\mathbb{D})$. Identifying $f$ and $\hat{f}$, we have the statement of the lemma

Remark. In general, every $f \in A$ is viewed as a function on the groupoid $G(c)=$ $G \times \mathbb{T}$. But, by Lemma 2.1 we can also view every $f \in A$ as a function on $G \times \mathbb{D}$, where $f((x, y), z), z \in \mathbb{D}$, is defined to be the value of the analytic extension of $f(x, y)$ at $z$.

Note now that according to the properties of crossed products we have:

(a) $\|u\|_{C^{*}(G(c), \sigma)}=\|u\|_{C^{*}(G, \sigma)}$ for every $u \in C^{*}(G, \sigma)$; that is, $C^{*}(G, \sigma)$ is isometrically embedded in $C^{*}(G(c), \sigma)$,

(b) $\left\|u \cdot e^{i n t}\right\|_{C^{*}(G(c), \sigma)}=\|u\|_{C^{*}(G, \sigma)}, \quad n \in \mathbb{N}, t \in \mathbb{R}, u \in C^{*}(G, \sigma)$.

Lemma 2.2. $C_{c}(G(c), \sigma) \cap A$ is dense in $A$.

Proof. By definition of $A$, the functions of the kind

$$
\sum_{n=0}^{k} u_{n}(x, y) e^{-i n t}, \quad u_{n} \in C^{*}(G, \sigma), \quad t \in \mathbb{R},
$$

are dense in $A$.

But also we know that $C_{c}(G, \sigma)$ is dense in $C^{*}(G, \sigma)$ by definition. So we can approximate every $u_{n}, n=1, \ldots, k$, in (2.1) by elements in $C_{c}(G, \sigma)$. In this case the functions we get will be in $C_{c}(G(c), \sigma) \cap A$, and we are done.

Lemma 2.3. Let $A$ be as above and $I \subseteq A$ a closed two-sided ideal. Define

$$
I(x, y)=\{f \in A(\mathbb{D}): F(x, y)=f, \text { for some } F \in I\} .
$$

Then $I(x, y)$ is a closed ideal in $A(\mathbb{D})$.

Proof. Note that we again refer to $A(\mathbb{D})$ as a subalgebra of $C(\mathbb{T})$ in a natural way.

Fix $(x, y) \in G$, and also fix a compact open $G$-set $\tau$ which contains $(x, y)$. Define

$$
\chi_{\tau}((x, y), t)=\left\{\begin{array}{ll}
1, & (x, y) \in \tau, \\
0, & (x, y) \notin \tau,
\end{array} \quad(x, y) \in G, \quad t \in \mathbb{T} .\right.
$$

Clearly, $\chi_{\tau} \in A$.

Now we will show that for any $\tau$ (compact, open $G$-set) and $s \in A(\mathbb{D}), s \chi_{\tau} \in A$, where

$$
s \chi_{\tau}((u, v), z)=s(z) \chi_{\tau}(u, v)
$$

If $s$ is an analytic trigonometric polynomial (i.e. $s=\sum_{m=0}^{n} a_{m} z^{m}$ ), then $s \chi_{\tau} \in A$ by the definition of $A$. Also it is clear that for every $s \in A(\mathbb{D}), s \chi_{\tau} \in C_{c}(G(c), \sigma)$.

Now fix an arbitrary $s \in A(\mathbb{D})$. Then we can find a sequence $\left\{s_{n}\right\} \in A(\mathbb{D})$ of analytic trigonometric polynomials such that $s_{n} \rightarrow s$ in the norm of $A(\mathbb{D})$. It is easy to see that $s_{n} \chi_{\tau} \rightarrow s \chi_{\tau}$ in the norm $\|\cdot\|_{I}$ of $C_{c}(G(c), \sigma)$. 
(Recall here, that for $f \in C_{c}(G(c), \sigma)$,

$$
\|f\|_{I}=\max \left\{\sup _{\substack{x \in G^{(0)} \\ t \in \mathbb{T}}} \sum_{y \in[x]}|f((x, y), t)|, \sup _{\substack{y \in G^{(0)} \\ t \in \mathbb{T}}} \sum_{x \in[y]}|f((y, x), c(x, y) t)|\right\}
$$

[R, p. 50].)

But $\|\cdot\| \leq\|\cdot\|_{I}$, where $\|\cdot\|$ is the usual norm of $C^{*}(G(c), \sigma)$. So $s_{n} \chi_{\tau} \rightarrow s \chi_{\tau}$ in norm. Hence $s \chi_{\tau} \in A$ for every $s \in A(\mathbb{D})$.

Now let $I \subseteq A$ be a two-sided closed ideal and $f \in I(x, y)$. Then there exists $F \in I$ such that $F(x, y)=f$. Hence $F \cdot\left(s \chi_{\tau}\right) \in I$ for an arbitrary compact open $G$-set as above and arbitrary $s \in A(\mathbb{D})$.

If now we have $(x, y) \in \tau$, then

$$
\begin{aligned}
F \cdot\left(s \chi_{\tau}\right)((x, y), z) & =\sum_{u \in[x]} F((x, u), z)\left(s \chi_{\tau}\right)((u, y), c(x, y) z) \cdot \sigma((x, u),(u, y)) \\
& =F(x, y) \cdot s=f \cdot s .
\end{aligned}
$$

But $A(\mathbb{D})$ is a commutative algebra and hence $I(x, y)$ is an ideal in $A(\mathbb{D})$.

Now we show that $I(x, y)$ is a closed ideal. First, for every $f \in A$ and compact open $G$-set $\tau$ we define the restriction $f \mid \tau$ : that is,

$$
(f \mid \tau)((u, v), z)= \begin{cases}f((u, v), z), & (u, v) \in \tau \\ 0, & \text { otherwise }\end{cases}
$$

It is clear that $f \mid \tau$ is in $A$. Moreover in the same way as in [MS, Prop. 3.6], we have that if $f \in I$, then $f \mid \tau \in I$ for every compact open $G$-set $\tau$ (all $h_{i}$ which are defined in [MS, Prop. 3.6] are in A.)

Now let $\left\{F_{n}\right\} \in I$ be such that $F_{n}(x, y) \rightarrow f$ for some $f \in A(\mathbb{D})$ in norm. We must find $F \in I$ such that $F(x, y)=f$.

Since $\left\{F_{n}(x, y)\right\}_{n=1}^{\infty}$ is a Cauchy sequence in $A(\mathbb{D})$, there exists a subsequence $\left\{F_{n_{k}}\right\}$ such that

$$
\left\|F_{n_{k}}(x, y)-F_{n_{k+1}}(x, y)\right\|_{A(\mathbb{D})}<\frac{1}{3 \cdot 2^{k+1}}, \quad k=1,2, \ldots
$$

Every function in $A$ is uniformly continuous on compacts, and hence we can choose a sequence of compact open $G$-sets $\tau_{k}$ such that $(x, y) \in \tau_{k}, k \in \mathbb{N}$ and

$$
\left\|F_{n_{k}}(u, v)-F_{n_{k}}(x, y)\right\|_{A(\mathbb{D})}<\frac{1}{3 \cdot 2^{k+1}}, \quad(u, v) \in \tau_{k} .
$$

Then from (3.1) and (3.2) it follows that

$$
\left\|F_{n_{k}}(u, v)-F_{n_{k+1}}(u, v)\right\|_{A(\mathbb{D})}<\frac{1}{2^{k+1}}, \quad(u, v) \in \tilde{\tau}_{k},
$$

where $\tilde{\tau}_{k}=\tau_{k} \cap \tau_{k+1}$.

Note also that we can choose $\left\{\tilde{\tau}_{k}\right\}$ to be a decreasing sequence (replace $\tilde{\tau}_{k}$ by $\left.\bigcap_{i=1}^{k} \tilde{\tau}_{i}\right)$. Now let us define a new sequence $s_{k} \in A$ by putting $s_{1}=F_{n_{1}} \mid \tilde{\tau}_{1}$, and $s_{k}=F_{n_{1}}\left|\left(\tilde{\tau}_{1} \backslash \tilde{\tau}_{2}\right)+F_{n_{2}}\right|\left(\tilde{\tau}_{2} \backslash \tilde{\tau}_{3}\right)+\cdots+F_{n_{k-1}}\left|\left(\tilde{\tau}_{k-1} \backslash \tilde{\tau}_{k}\right)+F_{n_{k}}\right| \tilde{\tau}_{n_{k}}$ for $k \geq 2$ (note here, that for $\tilde{\tau}_{i}, \tilde{\tau}_{i+1}$ compact open $G$-sets and $\tilde{\tau}_{i+1} \subseteq \tilde{\tau}_{i}$ we have that $\tilde{\tau}_{i} \backslash \tilde{\tau}_{i+1}$ is also a compact open $G$-set). Clearly (by the above observation), $\left\{s_{k}\right\} \in I$. Also it is clear that $s_{k}(x, y)=F_{n_{k}}(x, y)$. 
Now let us check that $\left\{s_{k}\right\}$ is a Cauchy sequence in the norm of $A$. Actually, it is enough to show that $\left\{s_{k}\right\}$ is a Cauchy sequence in the norm $\|\cdot\|_{I}$. First, note that from (3.3) it follows that

$$
\left\|s_{k}-s_{k+1}\right\|_{I}=\left\|\left(F_{n_{k}}-F_{n_{k+1}}\right) \mid \tilde{\tau}_{k+1}\right\|_{I}<\frac{1}{2^{k+1}}, \quad k \in \mathbb{N} .
$$

Now, take $\epsilon>0$ and choose $k, m$ such that $k>m$ and $2^{m}>\frac{1}{\epsilon}$.

Then, using (3.4), we have

$$
\begin{aligned}
\left\|s_{k}-s_{m}\right\|_{I} & \leq\left\|s_{m}-s_{m+1}\right\|_{I}+\left\|s_{m+1}-s_{m+2}\right\|_{I}+\cdots+\left\|s_{k-1}-s_{k}\right\|_{I} \\
& <\frac{1}{2^{m+1}}+\frac{1}{2^{m+2}}+\cdots+\frac{1}{2^{k}}<\frac{1}{2^{m}}<\epsilon .
\end{aligned}
$$

Then $\left\{s_{k}\right\}$ is a Cauchy sequence in $\|\cdot\|_{I}$ and thus in the norm of $A$. Let us denote by $s$ the limit of this sequence. $I$ is closed in norm, and hence $s \in I$. By [R, Prop. $4.2(\mathrm{II})] s_{k}(x, y) \rightarrow s(x, y)$ in the norm of $A(\mathbb{D})$. Hence $s(x, y)=f$.

Proposition 2.4. Let $f \in C_{c}(G(c), \sigma)$. If for every $(x, y) \in G, f(x, y) \in A(\mathbb{D})$, then $f \in A$.

Proof. Let $f \in C_{c}(G(c), \sigma)$ and $f(x, y) \in A(\mathbb{D}) \quad \forall(x, y) \in G$. Denote $\operatorname{supp} f=E$ and

$$
P_{G}(E)=\{(x, y) \in G: \exists t \in \mathbb{T}, \quad((x, y), t) \in E\} .
$$

Clearly, $P_{G}(E)$ is a compact set in $G$. First, consider the case when $P_{G}(E)$ is a compact open $G$-set. Given $\epsilon>0$ and $(x, y)$ in $P_{G}(E)$, there exists a compact open $\sigma$-set $\tau$ containing $(x, y)$ such that

$$
\|f(x, y)-f(u, v)\|_{A(\mathbb{D})}<\epsilon
$$

for every $(u, v)$ in $\tau$.

Since $P_{G}(E)$ is compact we can find a finite covering by such compact open $G$ sets $\left\{\tau_{i}\right\}_{i=1}^{n}$ in such a way that $P_{G}(E)=\bigcup_{i=1}^{n} \tau_{i}$ (because $P_{G}(E)$ now is a compact open $G$-set itself). Each set $\tau_{i}$ contains a point $\left(x_{i}, y_{i}\right)$ such that

$$
\left\|f\left(x_{i}, y_{i}\right)-f(u, v)\right\|_{A(\mathbb{D})}<\epsilon, \quad(u, v) \in \tau_{i} .
$$

Note here that we can choose a disjoint union because of the fact that intersections and differences of $G$-sets are also $G$-sets.

Define $F_{i}$ as follows:

$$
F_{i}((x, y), z)= \begin{cases}f\left(x_{i}, y_{i}\right), & (x, y) \in \tau_{i} \\ 0, & \text { otherwise }\end{cases}
$$

Since $f\left(x_{i}, y_{i}\right) \in A(\mathbb{D})$, then $F_{i} \in A$ (see the argument in the proof of Lemma 2.3 and note that $\left.F_{i}=f\left(x_{i}, y_{i}\right) \cdot \chi_{\tau_{i}}\right)$.

Define $F=\sum_{i=1}^{n} F_{i}$. So $F \in A$.

We will show that

$$
\|f-F\|<\epsilon \text {. }
$$

Actually, we will see that $\|f-F\|_{I}<\epsilon$. Since $P_{E}(G)$ is a $G$-set, for every $x \in X$ there exists not more then a unique point $y$ such that $(x, y) \in P_{G}(E)$. Hence for 
every fixed $x$ we have

$$
\begin{aligned}
& \sum_{y \in[x]}|(f-F)((x, y), t)|=\left|f((x, y), t)-F_{i}((x, y), t)\right| \\
& =\left|f((x, y), t)-f\left(\left(x_{i}, y_{i}\right), t\right)\right|<\epsilon, \quad t \in \mathbb{T} .
\end{aligned}
$$

Here $\tau_{i}$ is an unique $G$-set in the cover of $P_{G}(E)$ which contains $(x, y)$, and the above result follows from (4.2).

Hence

$$
\sup _{\substack{x \in G^{(0)} \\ t \in \mathbb{T}}} \sum_{y \in[x]}|(f-F)((x, y), t)| \leq \epsilon .
$$

Similarly we can see that

$$
\sup _{\substack{y \in G^{(0)} \\ t \in \mathbb{T}}} \sum_{x \in[y]}|(f-F)((y, x), c(x, y), t)| \leq \epsilon .
$$

Therefore $\|f-F\| \leq\|f-F\|_{I} \leq \epsilon$. So we can approximate $f$ by functions in $A$. Then $f \in A$. Now consider a general case for $P_{G}(E)$. Let us fix an arbitary cover of $P_{G}(E)$ by compact open $G$-sets (as above, we can choose a finite disjoint cover) such that

$$
P_{G}(E) \subseteq \bigcup_{i=1}^{k} \tilde{\tau}_{i} .
$$

Then we can write

$$
f=\sum_{i=1}^{k} f \mid \tilde{\tau}_{i} .
$$

But from our previous observation it is clear that $f \mid \tilde{\tau}_{i} \in A \quad \forall i=1, \ldots, k$. Then $f \in A$.

Proposition 2.5. Let $I \subseteq A$ be a two-sided closed ideal such that $I(x, y)=A(\mathbb{D})$ for every $(x, y) \in G$. Then $I=A$.

Proof. Fix $(x, y) \in G$. Since $I(x, y)=A(\mathbb{D})$, there exists $K \in I$ such that $K(x, y) \equiv$ 1. Let us choose $\epsilon>0$ and a compact open $G$-set $\tau$ which contains $(x, y)$ such that

$$
\|K(u, v)\|_{A(\mathbb{D})}>\epsilon, \quad \forall(u, v) \in \tau .
$$

Clearly this is possible because of the continuity of $K$. We can also choose $\tau$ such that for every $(u, v) \in \tau, K(u, v)$ is invertible. It is clear that the function $z \rightarrow(K((u, v), c(v, u) z))^{-1}$ is in $A(\mathbb{D}) \quad$ for all $(u, v) \in \tau$.

Then we can define $F \in A \cap C^{*}\left(G(c)^{0}, \sigma\right)$ as follows:

$$
F((v, v), z)= \begin{cases}K\left(\left(\tau^{-1}(v), v\right), c\left(v, \tau^{-1}(v)\right) z\right)^{-1}, & (v, v) \in d(\tau), \\ 0, & (v, v) \notin d(\tau) .\end{cases}
$$

Clearly $F$ is continuous and $\operatorname{supp} F \subseteq s(\tau) \times \mathbb{T}$. So $F \in C_{c}(G(c), \sigma)$ and then $F \in A$ by Proposition 2.4. It was proved in Lemma 2.3 that if $K \in I$ then $K \mid \tau \in I$. Then $(K \mid \tau) \cdot F \in I$. But it is easy to see that $(K \mid \tau) \cdot F=\chi_{\tau}$. 
Thus, for every $(x, y) \in G$ there exists compact open $G$-set $\tau_{x, y}$ such that $(x, y) \in$ $\tau_{x, y}$ and $\chi_{\tau_{x, y}} \in I$.

Now, let $F \in C_{c}(G(c), \sigma) \cap A$ be an arbitrary function. Denote $E=\operatorname{supp} F$, and as in Proposition 2.4 let $P_{G}(E)$ be the projection of $E$ on $G$. Consider

$$
r\left(P_{G}(E)\right)=\left\{(x, x) \in G^{(0)}, \quad(x, y) \in P_{G}(E)\right\} .
$$

Clearly $r\left(P_{G}(E)\right)$ is a compact set. Then it follows that there exists a finite covering of $r\left(P_{G}(E)\right)$ by compact open $G$-sets $\left\{\tau_{i}\right\}_{i=1}^{n} \in G^{(0)}$ such that $r\left(P_{G}(E)\right) \subseteq$ $\bigcup_{i=1}^{n} \tau_{i}$ and also $\chi_{\tau_{i}} \in I, i=1, \ldots, n$. It is easy to see that we can find a disjoint covering.

Then clearly $F=\sum_{i=1}^{n} \chi_{\tau_{i}} F$, and thus $F \in I$. Since this holds for every $F$ in $C_{c}(G(c), \sigma) \cap A$ and $I$ is a closed ideal, Lemma 2.2 completes the proof.

Lemma 2.6. In our previous notation, for an arbitrary $x \in X, y, z \in[x]$ we have

(1) $I(x, y)=I(x, z)$

(2) $I(x, y)=I(z, y) \circ c(x, z)$,

(3) $I(y, x)=I(x, y) \circ c(y, x)$,

(4) $I(y, y)=I(x, x) \circ c(y, x)$,

where $I(x, y)=I(u, v) \circ c(z, k)$ means that $f(t) \in I(u, v)$ iff $f(c(z, k) t) \in I(x, y)$.

Proof. (1) take $f \in I(x, y)$. Then there exists $F \in I$ such that $F(x, y)=f$. Now fix an arbitrary $z \in[x]$ and let $\tau$ be a compact open $G$-set containing $(y, z)$. Define $S=F \cdot \chi_{\tau}$. Then

$$
\begin{aligned}
& S((x, z), t)=\sum_{u \in[x]} F((x, u), t) \cdot \chi_{\tau}((u, z), c(x, u) t) \cdot \sigma((x, u),(u, z)) \\
& =F((x, y), t) \cdot \chi_{\tau}(y, z) \sigma((x, y),(y, z))=F((x, y), t) \cdot \sigma((x, y),(y, z))
\end{aligned}
$$

But clearly $S \in I$, and then $S(x, z)=F(x, y) \cdot \sigma((x, y),(y, z)) \in I(x, z)$. Thus $F(x, y)=f$ is in $I(x, z)$. So we have $I(x, y) \subseteq I(x, z)$. Due to the symmetry we have $I(x, y)=I(x, z)$.

(2) Let us take $F$ as in (1) and consider $H=\chi_{\tau} \cdot F$, where $\tau$ is a compact open $G$-set containing $(x, z)$. Then $H \in I$ and

$$
\begin{aligned}
& H((x, y), t)=\sum_{u \in[x]} \chi_{\tau}((x, u), t) \cdot F((u, y), c(x, u) t) \sigma((x, u),(u, y)) \\
& =F((z, y), c(x, z) t) \sigma((x, z),(z, y)) .
\end{aligned}
$$

Therefore $F((z, y), c(z, x) t) \in I(x, y)$. From this it follows

$$
I(z, y) \circ c(x, z) \subseteq I(x, y) .
$$

We use the fact that $c(x, z) \circ c(z, x)=1$ in order to obtain from (6.1)

$$
(I(z, y) \circ c(x, z)) \circ c(z, x) \subseteq I(z, y) \circ c(x, z) \subseteq I(x, y)
$$


(the last inclusion because of symmetry) or

$$
I(z, y) \subseteq I(x, y) \circ c(z, x) \subseteq I(z, y),
$$

and then

$$
\begin{aligned}
& I(x, y) \circ c(z, x)=I(z, y) \\
& I(z, y) \circ c(x, z)=I(x, y)
\end{aligned}
$$

(3) By (1) and (2) we have $I(y, x)=I(y, y)=I(x, y) \circ c(y, x)$.

(4) $I(y, y)=I(x, y) \circ c(y, x)=I(x, x) \circ c(y, x)$.

Proposition 2.7. Let $I \subseteq A$ be a maximal ideal. Then there exist $z_{0} \in \overline{\mathbb{D}}$ and $x \in X$ such that

$$
I=\left\{f \in A ; \quad f\left((u, v), c(x, u) z_{0}\right)=0, \quad u, v \in[x]\right\} .
$$

Proof. First, let us show that the set on the right hand side of (7.1) defines an ideal. Write $I_{0}$ for it.

Indeed, for $f \in I_{0}, g \in A$ we have

$$
\begin{aligned}
& f * g\left((u, v), c(x, u) z_{0}\right) \\
& =\sum_{k \in[x]} f\left((u, k), c(x, u) z_{0}\right) \cdot g\left((k, v), c(x, k) z_{0}\right) \sigma((u, k),(k, v))=0
\end{aligned}
$$

because $f\left((u, k), c(x, u) z_{0}\right)=0$ for every $k \in[x]$ by (7.1). Hence $f * g \in I_{0}$.

On the other hand, if $g \in I_{0}$ and $f \in A$ we have

$$
g\left((k, v), c(x, k) z_{0}\right)=0, \quad \forall k \in[x],
$$

by applying (7.1) for $g$. Hence $f * g \in I_{0}$ in this case too.

Therefore equation (7.1) defines an ideal. It is clearly a closed ideal, because the norm convergence implies pointwise convergence. Now let $I$ be a maximal ideal. Let us show that for every $f \in I$ there exist $z_{0} \in \overline{\mathbb{D}}$ and $x \in X$ such that $f$ is of the kind (7.1).

Indeed, by Lemma 2.3 and Proposition 2.5 we have that there exist $(x, y) \in G$ such that $I(x, y)$ is a proper ideal in $A(\mathbb{D})$, because $I \neq A$. Then there exists $z_{0} \in \overline{\mathbb{D}}$ such that $g\left(z_{0}\right)=0$ for every $g \in I(x, y)$ ([H, pp. 86-87]). But by Lemma 2.6 $I(u, v)=I(x, y) \circ c(u, x), u, v \in[x]$. Then for $f \in I(u, v), f\left(c(u, x) z_{0}\right) \equiv 0$. Hence $I \subseteq\left\{f \in A: f\left((u, v), c(x, u) z_{0}\right)=0, \quad u, v \in[x]\right\}$ (for the $x, z_{0}$ chosen above). By maximality of $I$ we have equality.

Now we introduce some more definitions. For a topological groupoid $G$ a subset $E \subseteq G^{(0)}$ is called invariant if $E=[E]$, where $[E]$ is a saturation of $E$, i.e $[E]=$ $r\left(d^{-1}(E)\right)$ (recall that $r(x)=x x^{-1}, d(x)=x x^{-1}$, for $x \in G$ ).

A topological groupoid $G$ is minimal if its range map $r: G \rightarrow G^{(0)}$ is open and if the only open invariant subsets of $G^{(0)}$ are the set $\emptyset$ and $G^{(0)}$ itself $([\mathrm{R}, \mathrm{p} .35])$.

From now on we assume that our groupoid $G$ is minimal. Now we define the asymptotic range (also called the essential range) of a cocycle $c$.

Let $G$ be a topological groupoid as above and $c: G \rightarrow \mathbb{T}$ a continuous cocycle. Then: 
(1) the range of $c$ is $R(c)=$ closure of $c(G)$,

(2) the asymptotic range of $c$ is $R_{\infty}(c)=\bigcap R\left(c_{U}\right)$, where the intersection is taken over all non-empty open subsets $U$ of $G^{(0)}$ and $c_{U}$ denotes the restriction of $c$ to $G \mid U$, where $G \mid U=\{x \in G, r(x) \in U, d(x) \in U\}$.

Moreover, if $u \in G^{(0)}$ then

(3) the range of $c$ at $u$ is $R^{u}(c)=$ closure of $c\left(G^{u}\right)$, where $G^{u}=r^{-1}(u)$,

(4) the asymptotic range of $c$ at $u$ is $R_{\infty}^{u}=\bigcap R^{u}\left(c_{U}\right)$, where the intersection is taken over a base of neighbourhoods of $u$.

Note also that by [R, Prop. 4.5(I)] $R_{\infty}(c)$ is a closed subgroup of $\mathbb{T}$, and therefore either $R_{\infty}(c)=\mathbb{T}$ or $R_{\infty}(c)=\left\{e^{i \frac{2 \pi k}{n}}, \quad k=0, \ldots, n-1\right\}$ for some $n \in \mathbb{N}$.

We shall now proceed to show that the maximal ideal structure of $A$ depends on the asymptotic range of $c$. The first result in this direction in the following theorem.

Theorem 2.8. Let $A=C^{*}(G(c), \sigma) \times_{\alpha} \mathbb{Z}_{+}$, as above. If $R_{\infty}(c)=\mathbb{T}$, then $A$ has a unique maximal ideal $I_{0}$.

Proof. Let $I$ be a maximal ideal in $A$. Then by Proposition 2.7 there exist $z_{0} \in \overline{\mathbb{D}}$ and $x \in X$ such that $f\left((u, v), c(x, u) z_{0}\right)=0, v, u \in[x]$, for every $f \in I$. Since $R_{\infty}(c)=\mathbb{T}$ and $G$ is minimal, then by [R, Corollary 4.16 (II)] $G(c)$ is minimal, and then every point $\left((x, x), t_{0}\right)$ in $(G(c))^{(0)}$ has a dense orbit (see also [R, Proposition 4.19 (II)]), i.e.:

$$
\overline{\left\{\left((u, u), c(x, u) t_{0}\right), \quad u \in[x]\right\}}=G^{(0)} \times \mathbb{T}
$$

Hence, if $z_{0} \in \mathbb{T}$, then for every $f \in I$ we have by continuity $f \mid G^{(0)} \times \mathbb{T}=0$. But by analyticity of $f(u, u)$ for every $(u, u) \in G^{(0)}$ we have then $f(u, u) \equiv 0$, and then $I(u, u)=\{0\}$ for every $(u, u) \in G^{(0)}$. But then by Lemma $2.6 I(u, k)=I(u, u)$, $k \in[u]$. Then it follows that $I=\{0\}$.

Assume now that $z_{0}$ is an interior point in $\mathbb{D}$. Then the same argument yields, for every $f \in I$, that $f\left|G^{(0)} \times \mathbb{T}\right| z_{0} \mid=0$, where $\mathbb{T}\left|z_{0}\right|$ is the circle with centre at the origin and radius $\left|z_{0}\right|$ (for the extension of $f$ to $\mathbb{D}$ ).

Actually, suppose $z_{0} \neq 0$. Write $t_{0}=z_{0} /\left|z_{0}\right|$ and let $((v, v), t) \in G^{(0)} \times \mathbb{T}$ be an arbitrary point. Then by (8.1) there exists a net $\left\{\left(\left(u_{\alpha}, u_{\alpha}\right), c\left(x, u_{\alpha}\right) t_{0}\right)\right\}$ such that $\left(\left(u_{\alpha}, u_{\alpha}\right), c\left(x, u_{\alpha}\right) t_{0}\right) \rightarrow((v, v), t)$. Then clearly $\left(\left(u_{\alpha}, u_{\alpha}\right), c\left(x, u_{\alpha}\right) z_{0}\right) \rightarrow$ $\left((v, v), t\left|z_{0}\right|\right)$. Hence if $f\left((u, u), c(x, u) z_{0}\right)=0, \quad u \in[x]$, then $f\left((v, v), t\left|z_{0}\right|\right)=$ 0 for every $(v, v) \in G^{(0)}$ and $t \in \mathbb{T}$, or $f\left|G^{(0)} \times \mathbb{T}\right| z_{0} \mid=0$.

By analyticity of $f(u, u)$ we obtain that $f(u, u)=0$, i.e. $I(u, u)=\{0\}$ for every $(u, u) \in G^{(0)}$, and therefore $I(u, v)=\{0\}$ for every $(u, v) \in G$. Hence $I=\{0\}$.

Now suppose $z_{0}=0$. Then, for every $f \in I$ we have $f((u, v), 0)=0, \quad u, v \in$ $[x]$. $G$ is a minimal groupoid, and therefore $\{(u, v), \quad u, v \in[x]\}$ is a dense set in $G$. By continuity of $f, f((s, p), 0)=0$ for every $(s, p) \in G$.

Define

$$
I_{0}=\{f \in A, \quad f((u, v), 0)=0, \quad(u, v) \in G\} .
$$


From our previous consideration it is clear that

$$
I_{0}=\{f \in A, \quad f((u, v), 0)=0 \text { for every }(u, v) \in G\},
$$

and then by Proposition $2.7 I_{0}$ is a maximal ideal. It follows from our proof that it is a unique maximal ideal in this case.

Now let us discuss some general facts about analytic crossed products. Let $A$ be a $C^{*}$-algebra and $B=A \times_{\alpha} \mathbb{Z}_{+}$the analytic crossed product of $A$ by $\alpha$. Denote:

$$
J_{1}=\overline{\left\{\sum_{n=1}^{\infty} a_{n} V^{n} ; \quad \sum_{n=1}^{\infty}\left\|a_{n}\right\|<\infty, \quad a_{n} \in A\right\}},
$$

where $V$ is a generator of $B$.

Proposition 2.9. If $A$ is simple, then $J_{1}$ is a maximal ideal in $B$.

Proof. It is easy to see that $J_{1}$ is an ideal in $B$ in any case. Now let $A$ be a simple Banach algebra and let $a \in B, a \notin J_{1}$. Denote by $I$ the ideal generated by $J_{1}$ and $a$.

By definition of $B$ there exists a sequence $\left\{f_{n}\right\} \in B$ of the following kind:

$$
\begin{gathered}
f_{n}=\sum_{m=0}^{\infty} u_{m}^{(n)} V^{m}, \quad u_{m}^{(n)} \in A, \quad \forall m, n \in \mathbb{N}, \text { such that } \sum_{m=0}^{\infty}\left\|u_{m}^{(n)}\right\|<+\infty, \\
f_{n} \rightarrow a \text { in the norm of } B .
\end{gathered}
$$

Note that there is a conditional expectation $P_{0}: A \times_{\alpha} \mathbb{Z} \rightarrow A$ satisfying

$$
P_{0}\left(\sum_{k=-n}^{n} a_{k} V^{k}\right)=a_{0}
$$

It is well known that $P_{0}$ is a projection of norm one and a positive map.

Then $\left\{u_{0}^{(n)}\right\}$ is a Cauchy sequence in $B$ (and in $A$ ). Denote by $u$ its limit. It is clear that $u \neq 0$, because $a \notin J_{1}$. Now define $\tilde{f}_{n}=\sum_{n=1}^{\infty} u_{m}^{(n)} V^{m}$. Clearly $\tilde{f}_{n} \in J_{1}$, $n \in \mathbb{N}$. Then $a-\tilde{f}_{n} \in I$. But $a-\tilde{f}_{n} \rightarrow u$. Hence $u \in I$. Since $A$ is simple, $A \subseteq I$ and by definition $J_{1} \subseteq I$. Then clearly $B=I$. Recall that $a$ was an arbitrary element in $B$ and therefore $J_{1}$ is a maximal ideal.

Now let $(A, \mathbb{Z}, \alpha)$ be, as usual, a $C^{*}$-dynamical $a$-system ( $a$ for "abelian"; see [Ped, p. 244 and pp. 267-268]). Define the dual system of $(A, \mathbb{Z}, \alpha)$, i.e. $\left(A \times_{\alpha}\right.$ $\mathbb{Z}, \mathbb{T}, \hat{\alpha})([$ Ped, Prop. 7.8.3]).

Denote by $\operatorname{sp}_{\hat{\alpha}}(a), a \in A \times{ }_{\alpha} \mathbb{Z}$, the Arveson spectrum of $a$ with respect to $\hat{\alpha}$ (see, for example [Ped, p. 303, 8.16]). Then, if as above $B=A \times_{\alpha} \mathbb{Z}_{+}$, we can write $B=\left(A \times_{\alpha} \mathbb{Z}\right)^{\hat{\alpha}}[0, \infty)$, that is, $B=\left\{a \in A \times_{\alpha} \mathbb{Z}, \operatorname{sp}_{\hat{\alpha}}(a) \subseteq[0, \infty)\right\}$, and similarly $J_{1}=\left(A \times_{\alpha} \mathbb{Z}\right)^{\hat{\alpha}}[1, \infty)$

Let $M\left(A \times_{\alpha} \mathbb{Z}\right)$ be the multiplier algebra of $A \times_{\alpha} \mathbb{Z}$.

For $V$ as above we have

$$
\begin{gathered}
b \cdot V^{n}=V^{n} \cdot \alpha^{n}(b), \\
\left(V^{n} \cdot b\right) \cdot V=V^{n+1} \cdot \alpha(b),
\end{gathered}
$$

and

for every $b \in A, n \in \mathbb{Z}$.

$$
V \cdot\left(V^{n} \cdot b\right)=V^{n+1} \cdot b
$$


It is clear that $V \in M\left(A \times_{\alpha} \mathbb{Z}\right)$ is a unitary element.

Lemma 2.10. $J_{1}=V \cdot B=\{V \cdot a, \quad a \in B\}$.

Proof. Clearly, $V \cdot B \subseteq J_{1}$. But $\operatorname{sp}_{\hat{\alpha}}(V)=\{1\}$ and $\operatorname{sp}_{\hat{\alpha}}\left(V^{*}\right)=\{-1\}$. Now take an arbitrary $b \in J_{1}$. Then $\operatorname{sp}_{\hat{\alpha}}(b) \subseteq[1, \infty)$. Then by [Ped, 8.3.3(II)] we have

$$
\operatorname{sp}_{\hat{\alpha}}\left(V^{*} b\right) \subseteq\{[1, \infty)\}+\{-1\}=[0, \infty),
$$

i.e. $V^{*} b \in B$. Then $b=V V^{*} b \in V \cdot B$.

Now we are ready to obtain our main result for the case $R_{\infty}(c)=\mathbb{T}$.

Theorem 2.11. Let $A, B, J_{1}$ be defined as above. If every two-sided closed ideal $I$ in $B$ is contained in $J_{1}$, then every such ideal is of the following kind: $J_{n}=V^{n} B$.

Proof. Note that from the conditions of the theorem it follows that $J_{1}$ is a unique maximal ideal in $B$.

Now let

$$
J_{n}=\overline{\left\{\sum_{k=n}^{\infty} u_{k} V^{k}, \quad u_{k} \in A, \quad \sum_{k=n}^{\infty}\left\|u_{k}\right\|<\infty\right\} .}
$$

Clearly, $J_{n}$ is an ideal in $B$. By the same argument as in Lemma 2.10 we can see that $J_{n}=V^{n} B$. Now let $I \subseteq B$ be an arbitrary ideal. Then $I \subseteq J_{1}$. Note that $\left\{J_{n}\right\}$ is a decreasing sequence of ideals. Now let $k$ be the minimal integer such that $J_{k}$ contains $I$, i.e. $I \subseteq J_{k}, I \nsubseteq J_{k+1}$. We will see that $I=J_{k}$.

Indeed, since , $I \subseteq J_{k}$, then for every $b \in I$ we have $b=V^{k} c, c \in B$. If $b \notin J_{k+1}$, then $c \notin J_{1}$. Let $g \in B$ be an arbitrary element. Then for $b$ as above we have

$$
b \cdot g=V^{k} \cdot c g \in I .
$$

Now if $h \in B$, it is easy to see that $V^{k} \cdot h\left(V^{*}\right)^{k} \in B$, and therefore we have

$$
V^{k} \cdot h\left(V^{*}\right)^{k} \cdot b=V^{k} h c \in I .
$$

From (11.1) and (11.2) we obtain that for some $c \notin J_{1}$ and arbitrary $h, g \in B$, $V^{k}(h c+c g) \in I$. Then $V^{k} \cdot J_{c} \subseteq I$, where $J_{c}$ is the ideal generated by $c$. But $c \notin J_{1}$ and hence $J_{c}=B$. Thus $I=J_{k}$.

Corollary 2.12. Let $A=C^{*}(G, \sigma) \times{ }_{\alpha} \mathbb{Z}_{+}$be the analytic crossed product of the $C^{*}$ algebra of an $r$-discrete, principal, minimal groupoid $G$ by $\alpha$ as above. If $R_{\infty}(c)=$ $\mathbb{T}$, then every ideal in $A$ is of the following kind:

$J_{n}=z^{n} \cdot A=\left\{f \in A ; f((x, y), z)=z^{n} \cdot g((x, y), z) \forall g \in A, \quad(x, y) \in G, z \in \overline{\mathbb{D}}\right\}$.

Proof. By [R, Prop. 4.5 (III), p. 101] and the fact that $G$ is minimal it follows that $C^{*}(G, \sigma)$ is simple. This is easy when $V=z \cdot I$, where $I$ is a unit of $M\left(C^{*}(G, \sigma) \times_{\alpha} \mathbb{Z}\right)$. By Theorem 2.8 we know that $I_{0}=J_{1}$ (in our recent notation) is a unique maximal ideal in this case, and using the argument of Proposition 2.7 and Theorem 2.8 we obtain that every ideal (closed, two-sided) $I$ in $A$ is contained in $J_{1}$. Hence we are in the conditions of the previous theorem, and everything follows from it.

Let us now study the case when $R_{\infty}(c)$ is a finite group.

Lemma 2.13. For a continuous cocycle $c$ of $G$, if $R_{\infty}(c)=\{1\}$, then there exists a continuous function $g: G \rightarrow \mathbb{T}$ such that $c(x, y)=g(y) \cdot g(x)^{-1},(x, y) \in G$. 
Proof. If $G^{(0)}$ is compact, then we are under the conditions of Theorem 4.10 of [R, p. 40], and our statement follows from that theorem.

In the general case, $G^{(0)}$ is a second countable locally compact Hausdorff space $\left(G^{(0)} \cong X\right)$; also, there exists a countable disjoint union of compact open subsets $\left\{\tau_{n}\right\}$ of $G^{(0)}$ such that

$$
G^{(0)}=\bigcup_{n=1}^{\infty} \tau_{n}
$$

Now consider for every $\tau_{n}$ the reduced groupoid

$$
G \mid \tau_{n}=\left\{x \in G ; \quad \tau(x), d(x) \in \tau_{n}\right\} .
$$

Then $R_{\infty}\left(c_{\tau_{n}}\right)=\{1\} \forall n \in \mathbb{N}$. Actually, by [R, Prop. 4.15, p. 44] $R_{\infty}(c)=R_{\infty}^{u}(c)$ for every $u \in G^{(0)}$. Fix some $n$, and fix $(u, u) \in G \mid \tau_{n}$. Clearly, by definition, $R_{\infty}^{u}(c) \supseteq R_{\infty}\left(c_{\left.\tau_{n}\right)}\right.$. But then $R_{\infty}\left(c_{\tau_{n}}\right)=\{1\}$ (actually, this is true for an arbitrary compact open $G$-set).

Now, for every $\tau_{n}$ we can use the above-mentioned Theorem 4.10 of [R, p. 40], i.e. for every $n$ we have $g_{n}$ such that $c(x, y)=g_{n}(y) \cdot g_{n}(x)^{-1}$ for every $(x, y) \in G \mid \tau_{n}$.

Let us define $g: G^{(0)} \rightarrow \mathbb{T}$. Clearly, every $g_{n}$ is defined up to a constant $\lambda \in \mathbb{T}$, i.e. we can replace $g_{n}$ by $\lambda g_{n}$ without changing $c(x, y)$ on $G \mid \tau_{n}$.

Now we set

$$
g(y)=c\left(x_{0}, y\right) \cdot g_{1}\left(x_{0}\right), \quad y \in\left[x_{0}\right] .
$$

Clearly, $g=g_{n}$ on $\tau_{n} \cap\left[x_{0}\right]$, and then by continuity we can extend $g(y)$, defined by formula (13.2), to all of $G^{(0)}$. It is clear that we obtain a well-defined, continuous function $g: G^{(0)} \rightarrow \mathbb{T}$ such that $c(x, y)=g(y) g(x)^{-1},(x, y) \in G$.

Lemma 2.14. Let $R_{\infty}(c)=\left\{e^{i \frac{2 \pi k}{n}}, \quad 0 \leq k \leq n-1\right\}$. Then there exists a continuous function $g: G^{(0)} \rightarrow \mathbb{T}$ such that $c^{n}(u, v)=g(v) g(u)^{-1}$ for every $(u, v) \in G$.

Proof. By the previous Lemma 2.13 it is sufficient to show that $R_{\infty}\left(c^{n}\right)=\{1\}$. Actually, by definition $R_{\infty}(c)=\bigcap_{U} R\left(c_{U}\right)$, where $U$ runs over all open non-empty subsets of $G^{(0)}$. Now if we fix some $U$ and consider the reduced groupoid $G \mid U$, then by [R, prop. 4.6 (I) p. 38 and prop. 4.15 p. 44] for this groupoid we obtain that if $a \in R\left(c_{U}\right)$ then also $a \cdot e^{i \frac{2 \pi k}{n}} \in R\left(c_{U}\right), 0 \leq k \leq n-1$.

Now let $e^{i \varphi_{0}} \in R_{\infty}\left(c^{n}\right)$. Then for an arbitrary open set $U \subseteq G^{(0)}$ we have $e^{i \varphi_{0}} \in$ $R_{\infty}\left(c^{n} \mid U\right)$, i.e. there exists a net $\left(x_{\alpha}, y_{\alpha}\right) \in G \mid U$ such that $c^{n}\left(x_{\alpha}, y_{\alpha}\right) \rightarrow e^{i \varphi_{0}}$, and therefore there exists a subnet $\left(x_{\alpha_{k}}, y_{\alpha_{k}}\right)$ such that $c\left(x_{\alpha_{k}}, y_{\alpha_{k}}\right) \rightarrow e^{\frac{i\left(\varphi_{0}+2 \pi m\right)}{n}}$ for some $m, 0 \leq m \leq n-1$. Then $e^{\frac{i\left(\varphi_{0}+2 \pi m\right)}{n}} \in R\left(c_{U}\right)$, and hence, as we have seen above, $e^{\frac{i\left(\varphi_{0}+2 \pi k\right)}{n}} \in R\left(c_{U}\right)$ for every $0 \leq k \leq n-1$. Since this is true for an arbitrary $U \subseteq G^{(0)}$, we have $e^{\frac{i\left(\varphi_{0}+2 \pi k\right)}{n}} \in R_{\infty}(c), 0 \leq k \leq n-1$. Then clearly $e^{i \varphi_{0}}=1$ and $R_{\infty}\left(c^{n}\right)=\{1\}$.

Define

$$
I_{0}=\{f \in A, \quad f((s, p), 0)=0, \quad(s, p) \in G\},
$$

and for $z_{0} \in \overline{\mathbb{D}} \backslash\{0\}$,

$$
I_{z_{0}}=\left\{f \in A, \quad f\left((s, p), \alpha_{i}^{(s)}\right)=0, \quad(s, p) \in G, \quad 1 \leq i \leq n\right\},
$$


where $\alpha_{i}^{(s)}$ are all the roots of the equation $z^{n}=g(s) z_{0}$, for a given function $g$ as in Lemma 2.14. (Recall that for $f \in A$ we have $f(x, y) \in A(\mathbb{D})$, where $A(\mathbb{D})$ is a subalgebra of $C(\mathbb{T})$, and by our definition $f((x, y), z)=f(x, y)(z)$ for $z \in A(\mathbb{D})$ (extension of $f(x, y)$ on $\mathbb{D})$.)

Theorem 2.15. Let $R_{\infty}(c)$ be a finite subgroup and $\left|R_{\infty}(c)\right|=n$. Then $I \subseteq A$ is a maximal ideal, $I=I_{z_{0}}$ for some $z_{0} \in \overline{\mathbb{D}}$.

Proof. By Proposition 2.7 with a little change of notation we have that if $I$ is a maximal ideal in $A$, then there exist $x \in X$ and $\tilde{z}_{0} \in \overline{\mathbb{D}}$ such that if $f \in I$ then

$$
f\left((u, v), c(x, u) \tilde{z}_{0}\right)=0, \quad \forall u, v \in[x] .
$$

If $\tilde{z}_{0}=0$ then by continuity of $f$ we have $f((s, p), 0)=0, \quad \forall(s, p) \in G$.

Actually, since $I$ is a maximal ideal, it follows from Proposition 2.9 that $I=J_{1}$, and we denote $I=I_{0}$ in this case.

Now assume $\tilde{z}_{0} \neq 0$. Note that since $I$ is a maximal ideal it is clear that every function of the kind (15.1) is in $I$; that is, the formula (15.1) is a criterion for the function $f$ to be in $I$ (see Proposition 2.7). Let us fix $(s, p) \in G$. Since $G$ is minimal, there exists a net $\left(u_{\alpha}, v_{\alpha}\right) \rightarrow(s, p)$ such that $u_{\alpha}, v_{\alpha} \in[x]$. Then $v_{\alpha} \rightarrow p$ in the topology of $G^{(0)}$, and $c\left(x, u_{\alpha}\right)=c\left(x, v_{\alpha}\right) \cdot c\left(v_{\alpha}, u_{\alpha}\right)$. By Lemma 2.14 there exists a continuous function $g: G^{(0)} \rightarrow \mathbb{T}$ such that $c^{n}\left(x, v_{\alpha}\right)=g(x)^{-1} g\left(v_{\alpha}\right)$, and then $c^{n}\left(x, v_{\alpha}\right) \rightarrow g(x)^{-1} g(p)$.

It follows that for the net $c\left(x, v_{\alpha}\right)$ there exist at least one and not more than $n$ limit points that roots of the equation $\alpha^{n}=g(x)^{-1} g(p)$.

Now choose a net $\left(u_{\alpha}, v_{\alpha}\right) \rightarrow(s, p)$ in such a way that there exists a limit for $c\left(x, v_{\alpha}\right)$, say $c\left(x, v_{\alpha}\right) \rightarrow a$ for some $a \in \mathbb{T}$. Let us show that we can find a net $\left(\tilde{u}_{\alpha}, \tilde{v}_{\alpha}\right)$ in such a way that $c\left(x, \tilde{v}_{\alpha}\right) \rightarrow a \cdot e^{i \frac{2 \pi k}{n}} \forall k, 0 \leq k \leq n-1$.

Actually, fix $\epsilon>0$ and choose $U_{s, p}$, a neighbourhood of $(s, p)$ (compact, open, $G$-set), such that $\left|c\left(x, v_{\alpha}\right)-a\right|<\frac{\epsilon}{2}$ for $v_{\alpha} \in U_{p}=s\left(U_{s, p}\right)$.

Now fix $v_{\alpha_{0}} \in U_{p}$ such that $v_{\alpha_{0}} \in[x]$ and

$$
\left|c\left(x, v_{\alpha_{0}}\right)-a\right|<\frac{\epsilon}{2} .
$$

By [R, Theorem 4.15, p. 44] we have $R_{\infty}(c)=R_{\infty}^{v_{\alpha_{0}}}(c)$. Then there exists $\tilde{v}_{\alpha_{0}} \in$ $U_{p} \cap[x]$ such that

$$
\left|c\left(v_{\alpha_{0}}, \tilde{v}_{\alpha_{0}}\right)-e^{i \frac{2 \pi k}{n}}\right|<\frac{\epsilon}{2}
$$

for an arbitrary fixed $k, 0 \leq k \leq n-1$.

Then by (15.2) and (15.3) we have

$$
\begin{aligned}
& \left|c\left(x, \tilde{v}_{\alpha_{0}}\right)-a \cdot e^{i \frac{2 \pi k}{n}}\right|=\left|c\left(x, v_{\alpha_{0}}\right) \cdot c\left(v_{\alpha_{0}}, \tilde{v}_{\alpha_{0}}\right)-a \cdot e^{i \frac{2 \pi k}{n}}\right| \\
& =\left|c\left(x, v_{\alpha_{0}}\right) \cdot a^{-1}-c\left(v_{\tilde{\alpha}_{0}}, v_{\alpha_{0}}\right) \cdot e^{i \frac{2 \pi k}{n}}\right| \\
& \leq\left|c\left(x, v_{\alpha_{0}}\right) \cdot a^{-1}-1\right|+\left|1-c\left(v_{\tilde{\alpha}_{0}}, v_{\alpha_{0}}\right) \cdot e^{i \frac{2 \pi k}{n}}\right| \\
& =\left|c\left(x, v_{\alpha_{0}}\right)-a\right|+\left|c\left(v_{\alpha_{0}}, v_{\tilde{\alpha}_{0}}\right)-e^{i \frac{2 \pi k}{n}}\right|<\epsilon .
\end{aligned}
$$

Since $U_{s, p}$ is a $G$-set, we have a unique $\tilde{u}_{\alpha_{0}} \in G^{(0)}$ such that $\left(\tilde{u}_{\alpha_{0}}, \tilde{v}_{\alpha_{0}}\right) \in U_{s, p}$. Therefore we can construct a net $\left(\tilde{u}_{\alpha}, \tilde{v}_{\alpha}\right) \rightarrow(s, p)$ such that $c\left(x, \tilde{v}_{\alpha}\right) \rightarrow a \cdot e^{i \frac{2 \pi k}{n}}$ for $0 \leq k \leq n-1$. 
Thus, we obtain that the set of limit points of the net $c\left(x, v_{\alpha}\right)$ is the set of all roots of the equation $\alpha^{n}=g(x)^{-1} \cdot g(p)$. But, as we have seen, $c\left(x, u_{\alpha}\right)=$ $c\left(x, v_{\alpha}\right) \cdot c\left(v_{\alpha}, u_{\alpha}\right)$ and $\left(u_{\alpha}, v_{\alpha}\right) \rightarrow(s, p)$. Then $c\left(v_{\alpha}, u_{\alpha}\right) \rightarrow c(p, s)$ by continuity of $c$. Hence, clearly the set of limit points of the net $c\left(x, u_{\alpha}\right)$ is the set of all roots of the equation $\alpha^{n}=g^{-1}(x) \cdot g(p) \cdot c^{n}(p, s)$. But $c^{n}(p, s)=g(p)^{-1} \cdot g(s)$, and so we have obtained the following statement: if $(s, p) \in G$ and $\left(u_{\alpha}, v_{\alpha}\right) \rightarrow(s, p)$ as above, then the set of all roots of the equation $\alpha^{n}=g^{-1}(x) \cdot g(s)$ is the set of all limit points of the set $\left\{c\left(x, v_{\alpha}\right), \quad v_{\alpha} \in[x]\right\}$.

Then for every function of the kind (15.1) we have the following statement by continuity:

$$
f\left((s, p), \alpha_{i}^{(s)}\right)=0 \quad \text { for every }(s, p) \in G
$$

and $\left\{\alpha_{i}^{(s)}\right\}_{i=1}^{n}$ is the set of all roots of the equation $\alpha^{n}=g(s) \cdot g(x)^{-1} \cdot \tilde{z}_{0}$.

As we have noted above, the functions of the kind (15.1) are exactly all the functions in the maximal ideal $I$, which is defined by $x$ and $\tilde{z}_{0}$. Now set $z_{0}=$ $g(x)^{-1} \cdot \tilde{z}_{0}^{n}$, and we obtain $I \subseteq I_{z_{0}}$. But clearly $I_{z_{0}} \subseteq I$. Hence $I=I_{z_{0}}$, and we are done.

Remark. In Proposition 2.7 we assigned to every maximal ideal a point in $X \times \overline{\mathbb{D}}$. This point is uniquely defined. However the point $z_{0}$, associated with the maximal ideal as in Theorem 2.15, is unique, as the following corollary shows.

Corollary 2.16. for $I_{z_{0}}$ as in the previous theorem we have:

(1) For every $(u, v) \in G$ there exists $f \in I_{z_{0}}$ such that $f((u, v), z) \neq 0$ if $z^{n} \neq g(u) z_{0}$

(2) $I_{z_{0}}=I_{z_{1}}$ iff $z_{0}=z_{1}$.

Proof. (1) Let $\tau$ be a compact open set such that $(u, v) \in \tau$, and define $f((x, y), z)$ $=\left(z^{n}-g(x) \cdot z_{0}\right) \cdot \chi_{\tau}(x, y)$. Clearly this function is in $I_{z_{0}}$, and it is the function we need.

(2) Clearly, if $z_{0}=z_{1}$ then $I_{z_{0}}=I z_{1}$.

Now, as in (1) define the same kind of functions for some compact open $\tau$-set:

$$
\begin{aligned}
& f_{0}((x, y), z)=\left(z^{n}-g(x) \cdot z_{0}\right) \cdot \chi_{\tau}(x, y), \\
& f_{1}((x, y), z)=\left(z^{n}-g(x) \cdot z_{1}\right) \cdot \chi_{\tau}(x, y) .
\end{aligned}
$$

Clearly, $f_{0} \in I_{z_{0}}, f_{1} \in I_{z_{1}}$ and it is easy to see that $f_{0} \notin I_{z_{1}}\left(f_{1} \notin I_{z_{0}}\right)$ if $z_{0} \neq z_{1}$.

Example 2.17. Let $X=\prod_{i=1}^{\infty} X_{i}, X_{i}=\{0,1\}$ for every $i \in \mathbb{N}$. Now let $G \subseteq X \times X$ be the equivalence relation such that $(x, y) \in G$ if $x(k)=y(k)$ for every $k \geq N$ for some $N \in \mathbb{N}$. Then $G$ has a natural groupoid structure. Also, let

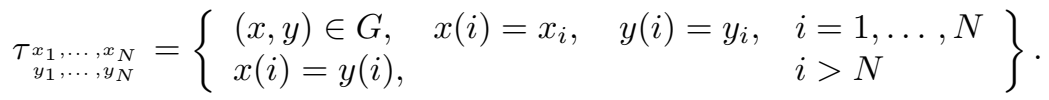

Define a topology on $G$ in such a way that every such set $\underset{x_{1}, \ldots, x_{N}}{\tau_{1}, \ldots, x_{N}}$ is a compact open set in $G$ (clearly, every such set is a $G$-set). Then it is easy to see that $G$ is an $r$-discrete principal minimal groupoid. 
Define $\tilde{c}: G \rightarrow \mathbb{Z}$ by $\tilde{c}(u, v)=\sum_{i}\left(u_{i}-v_{i}\right)$ for every $(u, v) \in G, u=(u(i))$, $v=(v(i))$. Then by $\left[\mathrm{R}\right.$, p. 130] we have $R_{\infty}(\tilde{c})=\mathbb{Z}$. Fix a number $\gamma \in \mathbb{R}$ and define $c: G \rightarrow \mathbb{T}$ as follows:

$$
c(u, v)=e^{i \tilde{c}(u, v) \gamma} .
$$

If, for example $\gamma=\frac{2 \pi}{n}$, then easily $R_{\infty}(c)=\left\{e^{\frac{2 \pi k}{n}}, \quad 0 \leq k \leq n-1\right\}$; and if $\gamma \neq \frac{m}{n} \pi$ for any $\frac{m}{n} \in \mathbb{Q}$ (for example $\gamma=e$ ), then $R_{\infty}(c)=\mathbb{T}$.

Analytic crossed products of the type we study here can arise as limit algebras of "nonselfadjoint circle algebras", as shown in the following example.

Example 2.18. Consider the case studied by J. Peters ([P1], [P2]). Here

$$
B=\lim _{\rightarrow}\left(A_{k}, \varphi_{k}\right)
$$

where $A_{k}=M_{n_{k}} \otimes A(\mathbb{D})$ is a tensor product of the disc algebra $A(\mathbb{D})$ and the algebra of $n_{k} \times n_{k}$ matrices and $\varphi_{k}: A_{k} \rightarrow A_{k+1}$ is a connecting homomorphism, defined as follows:

$$
\varphi_{k}(f)=\left(\begin{array}{ccc}
f \circ e^{i t_{1}^{k}} & & O \\
& \ddots & \\
O & & f \circ e^{i t_{\tau_{k}}^{k}}
\end{array}\right), \quad f \in A_{k},
$$

where $\tau_{k}=n_{k+1} / n_{k}$ and $t_{1}^{k}, \ldots, t_{\tau_{k}}^{k} \in \mathbb{R}$. Peters proved that in this case $B=$ $U \times_{\alpha} \mathbb{Z}_{+}$, where $U$ is a UHF-algebra $\left(U=\lim _{\rightarrow} M_{n_{k}}\right)$ and $\alpha: \mathbb{Z}_{+} \rightarrow$ Aut $U$ is a group homomorphism such that $\alpha(1)$ preserves the diagonal $D$ of $U$ elementwise $(D=$ $\lim _{\rightarrow} D_{n_{k}}$, where $D_{n_{k}}$ is a subalgebra of diagonal matrices in $\left.M_{n_{k}}\right)$, i.e. $\alpha(1)(f)=f$, $\overrightarrow{\forall f} \in D$.

Define $X=\prod_{i}^{\infty} X_{i}$, where $X_{i}=\left\{0,1, \ldots, \tau_{i}-1\right\}, i \geq 2$, and $X_{1}=\{0,1, \ldots$, $\left.n_{1}-1\right\}$. Define the groupoid $G$ and the topology on it similarly to what we did in Example 2.17. By [R, Theorem 115, p.134], $U \cong C^{*}(G)$. Also it follows from the definition of $\alpha$ that $\alpha$ is defined by some continuous cocycle $c: G \rightarrow \mathbb{T}$.

Since $B=C^{*}(G) \times_{\alpha} \mathbb{Z}_{+}$, we can use our previous results. Hence Theorem 2.15 gives us some generalization of the main theorem of Peters [P1, 4.13]. We will explain this point in detail later on.

Note also that Theorem 2.8 proves Peters conjecture (Remark 4.14) that when $\Gamma(\alpha)=\mathbb{T}$, there is a unique maximal ideal in $B$.

\section{Semisimplicity of $C^{*}(G, \sigma) \times_{\alpha} \mathbb{Z}_{+}$}

Now we want to prove that for $\alpha \in \operatorname{Aut}\left(C^{*}(G, \sigma)\right)$ as above, $A=C^{*}(G, \sigma) \times_{\alpha}$ $\mathbb{Z}_{+}$is always a semisimple Banach algebra.

Recall a few definitions. Let $U$ a Banach algebra and $a \rightarrow T a$ be a representation of $U$ on a linear space $X$, that is, $T: U \rightarrow L(X)$ is a homomorphism of $U$ into the algebra of all linear operators an $X$. A linear subspace $\mathcal{M}$ of $X$ is said to be invariant with respect to to $T$, if for every $a \in U, T a(\mathcal{M}) \subseteq \mathcal{M}$.

$T$ is strictly irreducible, if $\{0\}$ and $X$ are the only invariant subspaces for $T$.

The radical $R$ of an algebra $U$ is equal to the intersection of the kernels of all strictly irreducible representations of $U$. If $R=\{0\}$, then $U$ is said to be semisimple. Recall also the definition of a primitive ideal ([Rick, p. 53]): a 2-sided ideal is called primitive if it is the quotient of a maximal modular left ideal. 
Here the quotient of a left ideal $J$ in an algebra $U$ is the 2-sided ideal $J: U$ consisting of all $a \in U$ such that $a U \subseteq J$.

Lemma 3.1. If $R_{\infty}(c)$ is a finite subgroup of $\mathbb{T}$, then the intersection of all maximal ideals in $A$ is $\{0\}$.

Proof. Set $J=\bigcap_{z \in \overline{\mathbb{D}}} I_{z}$ (where $I_{z}$ is defined in Theorem 2.15). Let $f \in J$. Fix some point $\left((x, y), z_{0}\right) \in G \times \overline{\mathbb{D}}$ and set $\tilde{z}_{0}=z_{0}^{n} \cdot g(x)^{-1}$. Then $f \in I_{\tilde{z}_{0}}$. By Theorem 2.15 $f\left((x, y), z_{0}\right)=0$. Since this holds for every point in $G \times \overline{\mathbb{D}}, f((x, y), z) \equiv 0$.

Theorem 3.2. Let $A=C^{*}(G, \sigma) \times_{\alpha} \mathbb{Z}_{+}$, as above. Then $A$ is a semisimple algebra.

Proof. First, consider the case $R_{\infty}(c)=\mathbb{T}$. Denote, as above, by $R$ the radical of $A$. Then by Corollary 2.1, since $R$ is two-sided closed ideal, either $R=\{0\}$ or $R=V^{n} \cdot A$ for some $n \in \mathbb{N}$ (see the notation for Theorem 2.11). We know that

$$
R=\{b \in A, \quad \operatorname{sp}(a b)=\{0\}, \quad \forall a \in A\},
$$

where sp is the spectrum of an element in $A$.

Suppose $R \neq\{0\}$. Then there exists $n \in \mathbb{N}$ such that $R=V^{n} \cdot A$. Fix $\tau \subseteq G^{(0)}$, a compact open $G$-set. Then $\chi_{\tau} \in A$, and it is easy to see that $\chi_{\tau} \cdot\left(V^{n} \cdot \chi_{\tau}\right)=V^{n} \cdot \chi_{\tau}$. But clearly, $\operatorname{sp}\left(V^{n} \cdot \chi_{\tau}\right) \neq\{0\}$. Thus we have a contradiction. Hence $R=\{0\}$ and $A$ is semisimple.

Now consider the case when $R_{\infty}(c)$ is a finite group, i.e. $R_{\infty}(c)=\left\{e^{i \frac{2 \pi k}{m}}, \quad 0 \leq\right.$ $k \leq m-1\}$ for some $m \in \mathbb{N}$.

If there is a unit in $A$, then every maximal ideal is a modular ideal and hence primitive by [Rick, Theorem 2.29]. Then $R \subseteq \bigcap_{z \in \overline{\mathbb{D}}} I_{z}$.

But then by Lemma 3.1, $R=\{0\}$.

Suppose now that $A$ is a non-unital algebra. Clearly in this case the diagonal $G^{(0)}$ of $G$ is noncompact.

We show that in the case when $G^{(0)}$ is noncompact, every maximal ideal is nonmodular. Fix some $I_{z_{0}}, z_{0} \in \overline{\mathbb{D}}$. Suppose that $I_{z_{0}}$ is a modular ideal in $A$. Then there exists $e \in A$ such that

$$
f-e f, f-f \cdot e \in I_{z} \text { for every } f \in A \text {. }
$$

Fix a point $(s, s) \in G^{(0)}$, and let $\tau$ be a compact open $G$-set containing $(s, s)$. Then $\chi_{\tau} \in A$, and if we set $f=\chi_{\tau}$ we obtain

$$
\left(\chi_{\tau}-e \chi_{\tau}\right)\left((s, s), \alpha_{i}\right)=1-e\left((s, s), \alpha_{i}\right)=0,
$$

where $\left(\alpha_{i}\right)^{n}=g(s) \cdot z_{0}$. Then $e\left((s, s), \alpha_{i}\right)=1$, for every $1 \leq i \leq m$ and every $s \in G^{(0)}$. But by [R, Proposition $4.2(\mathrm{I})$, p. 99] $e \in C_{0}(G(c))$, and we obtain a contradiction. Hence $I_{z_{0}}$ is not a modular ideal (in particular, not left modular and not right modular).

We will show that for every maximal ideal $I_{z_{0}}$, in spite of this, there exists a left maximal modular ideal $J_{z_{0}}$ which contains $I_{z_{0}}$.

Let us fix a compact open $G$-set $\tau_{0} \subseteq G^{(0)}$. Then $G^{(0)} \backslash \tau_{0}$ is an open set. For every compact open $G$-set $\tau_{\alpha} \subseteq G^{(0)} \backslash \tau_{0}$ we define $\chi_{\tau_{\alpha}}$ and denote by $\Omega$ the left ideal generated by $\left\{\chi_{\tau_{\alpha}}\right\}_{\tau_{\alpha} \subseteq G^{(0)} \backslash \tau_{0}}$. Now check that for every $f \in A, f-f \chi_{\tau_{0}} \in \Omega$. First, 
fix $f \in C_{c}(G(c), \sigma) \cap A$. Denote $h=f-f \cdot \chi_{\tau_{0}}$. Then, clearly, $h \in C_{c}(G(c), \sigma) \cap A$. Denote also

$$
\tilde{G}=\left\{(y, y) \in G^{(0)}, \quad((x, y), t) \in \operatorname{supp} h \text { for some } t \in \mathbb{T} \text { and some } x \in[y]\right\} .
$$

Then $\tilde{G}$ is a compact in $G^{(0)}$.

If $(y, y) \in \tau_{0}$, then for every $t \in \mathbb{T}$ and $x \in[y]$ we have

$$
h((x, y), t)=f((x, y), t)-f \cdot \chi_{\tau_{0}}((x, y), t)=0 .
$$

Hence $\tilde{G} \subseteq G^{(0)} \backslash \tau_{0}$. Then there exists a finite covering of $\tilde{G}$ by compact open $G$-sets $\left\{\tau_{i}\right\}_{i=1}^{n}$ (clearly, we can choose disjoint sets) such that $\tilde{G} \subseteq \bigcup_{i=1}^{n} \tau_{i}$, and it is clear that

$$
h=\sum_{i=1}^{n} h \cdot \chi_{\tau_{i}} .
$$

Since every $\chi_{\tau_{i}}, i=1, \ldots, n$, is in $\Omega$ and $\Omega$ is a left ideal, then $h \in \Omega$.

Now let us take an arbitrary $f \in A$. Then by Lemma 2.2 there exists a sequence $f_{n} \in C_{c}(G(c), \sigma) \cap A$ such that $f_{n} \rightarrow f$ in the norm of $A$. Then $f_{n}-f_{n} \chi_{\tau_{0}} \rightarrow$ $f-f \chi_{\tau_{0}}$, and by our previous discussion and the fact that $\Omega$ is closed ideal, we obtain that $f-f \chi_{\tau_{0}} \in \Omega$. Hence $\Omega$ is a left modular ideal in $A$.

Now denote by $J$ the left closed ideal generated by $\Omega$ and $I_{z_{0}}$, for a fixed $z_{0} \in \overline{\mathbb{D}}$. First, let us show that $J \neq A$. For this, fix an arbitrary $(x, x) \in \tau_{0}$. Then there exists $z_{x} \in \overline{\mathbb{D}}$ such that $f\left((x, x), z_{x}\right)=0$ for every $f \in I_{z_{0}}$ (see Theorem 2.15).

Also $\chi_{\tau}\left((x, x), z_{x}\right)=0$ for every compact open set $\tau \in G^{(0)} \backslash \tau_{0}$; and then, clearly, $\left(g \cdot \chi_{\tau}\right)\left((x, x), z_{x}\right)=0$ for every $g \in A$. Then for every $h \in \Omega, h\left((x, x), z_{x}\right)$ $=0$, and then $f\left((x, x), z_{x}\right)=0$ for every $f \in J$. Hence $J \neq A$.

Denote by $J_{z_{0}}$ a maximal modular left ideal containing $J$. Then $I_{z_{0}} \subseteq J_{z_{0}}$, and clearly $I_{z_{0}}$ is the quotient of $J_{z_{0}}$. Then by our definition $I_{z_{0}}$ is a primitive ideal.

Therefore, also in this case $R \subseteq \bigcap_{z_{0} \in \overline{\mathbb{D}}} I_{z_{0}}$, and hence by Lemma $3.1 R=\{0\}$.

\section{A topological Description of the maXimal ideal SPACE of $C^{*}(G(c), \sigma) \times_{\alpha} \mathbb{Z}_{+}$FOR FINITE $R_{\infty}(c)$}

Here we shall prove that if $R_{\infty}(c)$ is finite, then the maximal ideal space $\mathcal{M}$ is homeomorphic to $\overline{\mathbb{D}} / R_{\infty}(c)$ (where we have the hull kernel topology on $\mathcal{M}$ and also the hull kernel topology of $A(\mathbb{D})$ on $\overline{\mathbb{D}}$ ). Recall that, by Theorem 2.15 , as a set, $\mathcal{M}=\left\{I_{z},: z \in \overline{\mathbb{D}}\right\}$. We start with the following lemma:

Lemma 4.1. (1) If $I_{z_{0}}$ is a maximal ideal of $A$, then for every $(x, y) \in G$ we have

$$
I_{z_{0}}(x, y)=\bigcap_{i=1}^{n} J_{\alpha_{i}^{x}}
$$

where $\left\{\alpha_{i}^{x}\right\}_{i=1}^{n}$ are all the roots of the equation $\left(\alpha_{i}^{x}\right)^{n}=g(x) z_{0}$ and

$$
J_{\alpha_{i}^{x}}=\left\{f \in A(\mathbb{D}): f\left(\alpha_{i}^{x}\right)=0\right\}
$$

is a maximal ideal in $A(\mathbb{D})$. 
(2) For any subset $\mathcal{K} \subseteq \mathcal{M}$ we have

$$
\left(\bigcap_{I_{z} \in \mathcal{K}} I_{z}\right)(x, y)=\bigcap_{I_{z} \in \mathcal{K}} I_{z}(x, y)
$$

for every $(x, y) \in G$.

Proof. (1) Let $I_{z_{0}}$ be a maximal ideal in $A$. Then $I_{z_{0}}(x, y)$ is a closed ideal in $A(\mathbb{D})$ (see Lemma 2.3) such that for every $f$ in $I_{z_{0}}(x, y), f\left(\alpha_{i}^{x}\right)=0$ for $\alpha_{i}^{x}$ that satisfy $\left(\alpha_{i}^{x}\right)^{n}=g(x) z_{0}, i=1, \ldots, n$ (where $n$ is an order of $R_{\infty}(c)$ ) (see Theorem 2.15).

Then $I_{z_{0}}(x, y) \subseteq \bigcap_{i=1}^{n} J_{\alpha_{i}^{x}}$. Now we will show that we have an equality.

Actually, by [H, p. 88], $z-\alpha_{i}^{x}$ generates $J_{\alpha_{i}^{x}}$. Then it is easy to see that $\prod_{i=1}^{n}\left(z-\alpha_{i}^{x}\right)$ generates $\bigcap_{i=1}^{n} J_{\alpha_{i}^{x}}$.

Indeed, let us check that $\left(z-\alpha_{1}^{x}\right)\left(z-\alpha_{2}^{x}\right)$ generates $J=\bigcap_{i=1}^{2} J_{\alpha_{i}^{x}}$. Fix an arbitrary $f \in J$. Then there exists a sequence $\left\{f_{n}\right\} \in A(\mathbb{D})$ such that $\left(z-\alpha_{1}^{x}\right) f_{n} \stackrel{\|\cdot\|_{A(\mathbb{D})}}{\longrightarrow} f$. But $f\left(\alpha_{2}^{x}\right)=0$. Then, clearly, $\left(z-\alpha_{1}^{x}\right)\left(f_{n}-f_{n}\left(\alpha_{2}^{x}\right)\right) \stackrel{\|\cdot\|_{A(\mathbb{D})}}{\longrightarrow} f$. But $f_{n}-f_{n}\left(\alpha_{2}^{x}\right) \in J_{\alpha_{2}^{x}}$ and we can choose a sequence $\left\{g_{n}\right\} \in A(\mathbb{D})$ such that

$$
\left\|\left(z-\alpha_{2}^{x}\right) \cdot g_{n}-\left(f_{n}-f_{n}\left(\alpha_{2}^{x}\right)\right)\right\|<\frac{1}{n}
$$

and then, clearly, $\left(z-\alpha_{1}^{x}\right)\left(z-\alpha_{2}^{x}\right) g_{n} \stackrel{\|\cdot\|_{A(\mathbb{D})}}{\longrightarrow} f$, or in other words, $\left(z-\alpha_{1}^{x}\right)\left(z-\alpha_{2}^{x}\right)$ generates $J_{\alpha_{1}^{x}} \cap J_{\alpha_{2}^{x}}$. By induction it is clear that $\prod_{i=1}^{n}\left(z-\alpha_{i}^{x}\right)$ generates $\bigcap_{i=1}^{n} J_{\alpha_{i}^{x}}$. Note that in our case $\prod_{i=1}^{n}\left(z-\alpha_{i}^{x}\right)=z^{n}-g(x) z_{0}$.

Now define $F$ in $A$ as follows:

$$
F((u, v), z)=\left(z^{n}-g(u) z_{o}\right) \chi_{\tau}(u, v)
$$

(for some compact open $G$-set $\tau$ in $G$ such that $(x, y) \in \tau$ ). Then it is clear that $F$ lies in $I_{z_{0}}$ and $F((x, y), z)=z^{n}-g(x) z_{0}$. Then $z^{n}-g(x) z_{0} \in I_{z_{0}}(x, y)$, and hence we have

$$
I_{z_{0}}(x, y)=\bigcap_{i=1}^{n} J_{\alpha_{i}^{x}} .
$$

(2) Let $\mathcal{K} \subseteq \mathcal{M}$ be an arbitrary subset. Then, clearly,

$$
\bigcap_{I_{z} \in \mathcal{K}} I_{z}(x, y) \supseteq\left(\bigcap_{I_{z} \in \mathcal{K}} I_{z}\right)(x, y)
$$

for every $(x, y) \in G$. We want to prove the inverse inclusion.

As we know $\bigcap_{I z \in \mathcal{K}} I_{z}(x, y)$ is a closed ideal in $A(\mathbb{D})$. Then (by [H, p. 88] there exists $f(z) \in A(\mathbb{D})$ which generates this ideal. It remains to prove that

$$
f(z) \in\left(\bigcap_{I z \in \mathcal{K}} I_{z}\right)(x, y)
$$

Since $g: G^{0} \rightarrow \mathbb{T}$ is a continuous function we can find a compact open neighbourhood $\tau$ of $(x, y)$ such that a function $s(u)=\sqrt[n]{g(x) g^{-1}(u)}$ will be well defined as a continuous function on $r(\tau)$, where $r(\tau)=\left\{(u, u) \in G^{0}, \quad(u, v) \in \tau\right\}$ and $s(x)=1$. Then we can define $P \in A$ as follows:

$$
P((u, v), z)=f(s(u) \cdot z) \chi_{\tau}(u, v) .
$$

It is easy to see that $P \in \bigcap_{I z \in \mathcal{K}} I_{z}$ (by the definition of $f(z)$ ) and also $P(x, y)=f$. So we are done. 
Theorem 4.2. Let $\mathcal{M}$ be a maximal ideal space of $A=C^{*}\left(G(G, \sigma) \times_{\alpha} \mathbb{Z}_{+}\right.$and $R_{\infty}(c)$ a finite subgroup of $\mathbb{T}$. Then $\mathcal{M}$ with the hull-kernel topology is homeomorphic to $\overline{\mathbb{D}} / R_{\infty}(c)$, where $\overline{\mathbb{D}}$ is provided with the hull-kernel topology of $A(\mathbb{D})$.

Proof. Define $\varphi: \mathcal{M} \rightarrow \overline{\mathbb{D}} / R_{\infty}(c)$ as follows: $\varphi\left(I_{z_{0}}\right)=\left[z_{0}\right]=\left\{\sqrt[n]{z_{0}}\right\}_{i=1}^{n}$. It is clear that this $\varphi$ is a one-to-one transformation. Now we have to check that $\varphi$ and $\varphi^{-1}$ are continuous.

Let $\mathcal{K} \subseteq \mathcal{M}$ be a closed set. Then we must show that $\varphi(\mathcal{K})$ is a closed set in $\overline{\mathbb{D}} / R_{\infty}(c)$, or, more precisely

If for $\left[z_{i}\right] \in \overline{\mathbb{D}} / R_{\infty}(c)$

$$
J_{\left[z_{0}\right]} \supseteq \bigcap_{[z] \in \varphi(\mathcal{K})} J_{[z]}
$$

then $\left[z_{0}\right] \in \varphi(\mathcal{K})$, where $J_{[z]}=\bigcap_{i=1}^{n} J_{\alpha_{i}^{z}}$ for $\left\{\alpha_{i}^{z}\right\}_{i=1}^{n}$ are all the roots of the equation $\left(\alpha_{i}^{z}\right)^{n}=z$ and $J_{\alpha_{i}^{z}}=\left\{f \in A(\mathbb{D}), \quad f\left(\alpha_{i}^{z}\right)=0\right\}$ is a maximal ideal in $A(\mathbb{D})$ as above.

Let us agree to identify $z$ and $I_{z}$ for every $z \in \mathcal{M}$.

We want to check that $I_{z_{0}} \supseteq \bigcap_{z \in \mathcal{K}} I_{z}$, where $z_{0}=\varphi^{-1}\left(\left[z_{0}\right]\right)$.

Actually, it was shown in Lemma 4.1 that for every $(x, y) \in G, I_{z_{0}}(x, y)=$ $J_{\left[g(x) z_{0}\right]}$. But then by $(2.1)$ we clearly have that

$$
J_{\left[g(x) z_{0}\right]} \supseteq \bigcap_{[z] \in \varphi(\mathcal{K})} J_{[g(x) z]} .
$$

Then

$$
I_{z_{0}}(x, y) \supseteq \bigcap_{z \in \mathcal{K}} I_{z}(x, y)=\left(\bigcap_{z \in \mathcal{K}} I_{z}\right)(x, y)
$$

for every $(x, y) \in G$.

The last equality holds by the previous lemma. But then from (2.2) it follows that for every $f \in\left(\bigcap_{z \in \mathcal{K}} I_{z}\right)$ and for every $(x, y) \in G, f(x, y) \in I_{z_{0}}(x, y)$, i.e. $f\left((x, y), \alpha_{i}^{x}\right)=0$ for every $\alpha_{i}^{x}$ such that $\left(\alpha_{i}^{x}\right)^{n}=g(x) z_{0}$.

Then $f \in I_{z_{0}}$, and we obtain the inclusion

$$
\left(\bigcap_{z \in \mathcal{K}} I_{z}\right) \subseteq I_{z_{0}}
$$

But $\mathcal{K}$ is a closed set in $\mathcal{M}$ in the hull-kernel topology, and hence $z_{0} \in \mathcal{K}$. Therefore, $\left[z_{0}\right] \in \varphi(\mathcal{K})$ and $\varphi(\mathcal{K})$ is closed in $\overline{\mathbb{D}} / R_{\infty}(c)$.

Conversely, let $S \subseteq \overline{\mathbb{D}} / R_{\infty}(c)$ be a closed set. Then we must check that $\varphi^{-1}(S)$ is a closed set in $\mathcal{M}$. So take $z_{0} \in \mathcal{M}$ satisfying

$$
I_{z_{0}} \supseteq \bigcap_{z \in \varphi^{-1}(S)} I_{z} \text {. }
$$

Then by Lemma 4.1 we have, for every $(x, y)$ in $G$,

$$
I_{z_{0}}(x, y) \supseteq\left(\bigcap_{z \in \varphi^{-1}(S)} I_{z}\right)(x, y)=\bigcap_{z \in \varphi^{-1}(S)} I_{z}(x, y) .
$$

But then $J_{\left[g(x) z_{0}\right]} \supseteq \bigcap_{[z] \in S} J_{[g(x) z]}$, and it follows that $J_{\left[z_{0}\right]} \supseteq \bigcap_{[z] \in S} J_{[z]}$.

$S$ is closed, and therefore $\left[z_{0}\right] \in S$. Then $z_{0} \in \varphi^{-1}(S)$, and thus $\varphi^{-1}(S)$ is closed. Hence $\varphi$ is a homeomorphism. 


\section{The ASYMPtotic RANGe AS AN INVARIANT WITH RESPECT TO ISOMETRIC ISOMORPHISM}

In this section we prove one important feature of the asymptotic range.

Theorem 5.1. Let $A_{1}=C^{*}(G, \sigma) \times{ }_{\alpha_{1}} \mathbb{Z}_{+}$and $A_{2}=C^{*}(G, \sigma) \times_{\alpha_{2}} \mathbb{Z}_{+}$, where $\alpha_{1}$ and $\alpha_{2}$ are automorphisms which correspond to cocycles $c_{1}$ and $c_{2}$, and $G$ is defined as above ( $r$-discrete, principal, minimal, amenable groupoid). Then, if $A_{1}$ and $A_{2}$ are isometrically isomorphic, their asymptotic ranges coincide, i.e. $R_{\infty}\left(c_{1}\right)=$ $R_{\infty}\left(c_{2}\right)$.

Proof. Let $\varphi: A_{1} \rightarrow A_{2}$ be a given isometric isomorphism. Since $A_{1}$ and $A_{2}$ are isomorphic we have a one-to-one correspondence between their maximal ideals.

Now, if $R_{\infty}\left(c_{1}\right)=\mathbb{T}$, we have a unique maximal ideal by Theorem 2.8 , and hence by symmetry we obtain that $R_{\infty}\left(c_{1}\right)=\mathbb{T}$ iff $R_{\infty}\left(c_{2}\right)=\mathbb{T}$.

Now let $R_{\infty}\left(c_{1}\right)$ and $R_{\infty}\left(c_{2}\right)$ be finite closed subgroups of $\mathbb{T}$ such that $\left|R_{\infty}\left(c_{1}\right)\right|$ $=m$ and $\left|R_{\infty}\left(c_{2}\right)\right|=n$.

If $m=n$, we are done. Now suppose that $n>m$ (in particular, $n>1$ ). We show that in this case $\varphi\left(z \cdot A_{1}\right)=z \cdot A_{2}$. Indeed, suppose not. Then, if $g_{1}$ and $g_{2}$ from $G^{(0)}$ to $\mathbb{T}$ are the continuous functions, which correspond to $c_{1}, c_{2}$ by Lemma 2.14 , according to Theorem 2.15 there exists $z_{0} \in \overline{\mathbb{D}}$ such that $\varphi\left(z \cdot A_{1}\right)=I_{z_{0}}$, where

$$
I_{z_{0}}=\left\{f \in A_{2}, \quad f\left((u, v), \alpha_{i}^{(u)}\right)=0, \quad 1 \leq i \leq n, \quad \text { for every }(u, v) \in G\right\},
$$

where $\left\{\alpha_{i}^{(u)}\right\}_{i=1}^{n}$ are all the roots of the equation $z^{n}=g_{2}(u) z_{0}$.

Note that every $f \in A_{1}$ can be written as follows: $f=f_{1}+f_{2}$, where $f_{1}=$ $f((u, v), z)-f((u, v), 0)$ is in $z \cdot A_{1}$ and $f_{2}=f((u, v), 0)$ for every $(u, v) \in G$. Clearly $f_{2} \in C^{*}(G, \sigma)$. By $[\mathrm{AS}]$ we know that $\varphi\left(C^{*}(G, \sigma)\right)=C^{*}(G, \sigma)$, since $C^{*}(G, \sigma)=A_{1}^{*} \cap A_{1}=A_{2}^{*} \cap A_{2}$. Then, from the previous observation and the fact that $\varphi\left(z \cdot A_{1}\right)=I_{z_{0}}$, it follows that every function $g$ in $A_{2}$ can be represented in $g=g_{1}+g_{2}$, where $g_{1} \in I_{z_{0}}, g_{2} \in C^{*}(G, \sigma)$.

Now we fix some $G$-set (compact, open) $\chi_{\tau}$ and consider $z \cdot \chi_{\tau} \in A_{2}$. As we have seen in Lemma $4.1, z^{n}-g(u) z_{0}$ generates the ideal $I_{z_{0}}(u, v) \subseteq A(\mathbb{D})$ for every $(u, v) \in G$, and then clearly $z \cdot \chi_{\tau}$ cannot be represented as above. Then we have a contradiction. Hence $\varphi\left(z \cdot A_{1}\right)=z \cdot A_{2}$.

Now we prove that $\varphi\left(z^{k} \cdot A_{1}\right)=z^{k} \cdot A_{2}$, for every $k \in \mathbb{N}$. We will start with $F$ in $C_{c}\left(C^{*}(G, \sigma)\right) \cap A_{1}$. Using the same argument as in Proposition 2.5, we can write $F=\sum_{i=1}^{n} \chi_{\tau_{i}} F$, where $\tau_{i} \subseteq G^{(0)}, i=1, \ldots, n$, are compact open sets (disjoint). Also, it is clear that $\left(z \cdot \chi_{\tau_{i}}\right)^{k}=z^{k} \cdot \chi_{\tau_{i}}$.

Now we have

$$
\begin{gathered}
\varphi\left(z^{k} \cdot F\right)=\varphi\left(z^{k} \cdot \sum_{i=1}^{n} \chi_{\tau_{i}} F\right)=\varphi\left(\left(\sum_{i=1}^{n} z^{k} \cdot \chi_{\tau_{i}}\right) \cdot F\right) \\
=\sum_{i=1}^{n} \varphi\left(z^{k} \cdot \chi_{\tau_{i}}\right) \cdot \varphi(F)=\sum_{i=1}^{n} \varphi\left(\left(z \cdot \chi_{\tau_{i}}\right)^{k}\right) \cdot \varphi(F)=\sum_{i=1}^{n}\left(\varphi\left(z \cdot \chi_{\tau_{i}}\right)\right)^{k} \cdot \varphi(F) .
\end{gathered}
$$

Since $\varphi\left(z \cdot \chi_{\tau_{i}}\right)$ is in $z \cdot A_{2}$, then clearly $\left(\varphi\left(z \cdot \chi_{\tau_{i}}\right)\right)^{k}$ is in $z^{k} \cdot A_{2}$. Hence $\varphi\left(z^{k} F\right) \subseteq$ $z^{k} \cdot A_{2}$ for every $F$ in $C_{c}(G(c), \sigma) \cap A_{1}$. By the density of the functions of this 
kind in $A_{1}$ (Lemma 2.2) we obtain $\varphi\left(z^{k} A_{1}\right) \subseteq z^{k} \cdot A_{2}$. Finally, by symmetry we have $\varphi\left(z^{k} A_{1}\right)=z^{k} \cdot A_{2}$ for any $k \in \mathbb{N}$.

Now fix some compact open $G$-set $\tau$ and denote

$$
P=\varphi\left(z^{m} \cdot \chi_{\tau}\right), \quad V=\varphi\left(g_{1}(u) \chi_{\tau}(u, v)\right) .
$$

If $z_{0} \in \overline{\mathbb{D}}, z_{0} \neq 0$, then $\varphi\left(I_{z_{0}}\right)=I_{\tilde{z}_{0}}$ for some $\tilde{z}_{0} \in \overline{\mathbb{D}}, \tilde{z}_{0} \neq 0$ (as we have proven), and

$$
\varphi\left(\left(z^{m}-z_{0} g_{1}\right) \cdot \chi_{\tau}\right)((x, y), z)=\left(P-z_{0} V\right)((x, y), z) \in I_{\tilde{z}_{0}} .
$$

Write $h_{\tilde{z}_{0}}=P-z_{0} V$. Then $h_{\tilde{z}_{0}} \in I_{\tilde{z}_{0}}$.

As we have seen in Lemma $4.1, z^{n}-\tilde{z}_{0} g_{2}(x)$ generates the ideal $I_{\tilde{z}_{0}}(x, y) \subseteq A(\mathbb{D})$. Therefore

$$
h_{\tilde{z}_{0}}((x, y), z)=\left(z^{n}-\tilde{z}_{0} g_{2}(x)\right) \cdot f_{(x, y), \tilde{z}_{0}}(z)
$$

for any $(x, y) \in G, z \in S_{R}(0)$ and $f_{(x, y), z_{0}} \in A\left(S_{R}(0)\right)$ for some $R<1$.

Here $S_{R}(0)=\{z \in \mathbb{C}, \quad|z| \leq R\}$, and $A\left(S_{R}(0)\right)$ is defined in the same way as $A(\mathbb{D})$.

In fact, fix an arbitrary $0<R<1$. If $\left|\tilde{z}_{0}\right|<1$, our statement follows from the first theorem of Hoffman [H, p. 66]. If $\left|\tilde{z}_{0}\right|=1$ then $z^{n}-\tilde{z}_{0} g_{2}(x)$ (for fixed $x \in X$ ) has no zeroes on $S_{R}(0)$. Then we set $f_{(x, y), \tilde{z}_{0}}(z)=h_{\tilde{z}_{0}}((x, y), z) \cdot\left(z^{n}-\tilde{z}_{0} g_{2}(x)\right)^{-1}$, and clearly $f_{(x, y), \tilde{z}_{0}}$ is analytic on $S_{R}(0)$, because $h_{\tilde{z}_{0}}(x, y)$ is in $A(\mathbb{D})$.

From (5.1) we obtain that, for every pair $z_{0}$ and $\tilde{z}_{0}$,

$$
P=h_{\tilde{z}_{0}}+\tilde{z}_{0} V .
$$

We will show that for every fixed $(x, y) \in G$ we have $P((x, y), z)=p_{(x, y)}\left(z^{n}\right)$ for some $p_{(x, y)}(z)$ in $A\left(S_{R}(0)\right)$.

Indeed, fix $(x, y) \in G$ and $\alpha_{1}, \alpha_{2}$ in $S_{R}(0)$ with $\alpha_{1}^{n}=\alpha_{2}^{n}$. Write $x_{0}=\alpha_{1}^{n}=\alpha_{2}^{n}$ and $\tilde{z}_{0}=\frac{x_{0}}{g_{2}(x)}$.

Note that we clearly have a one-to-one correspondence between $z_{0}$ and $\tilde{z}_{0}$.

Then by $(5.2) h_{\tilde{z}_{0}}\left((x, y), \alpha_{1}\right)=h_{\tilde{z}_{0}}\left((x, y), \alpha_{2}\right)=0$ and $V\left((x, y), \alpha_{1}\right)=$ $V\left((x, y), \alpha_{2}\right)$, because $V$ is in $C^{*}(G, \sigma)$. Hence by (5.3)

$$
P\left((x, y), \alpha_{1}\right)=P\left((x, y), \alpha_{2}\right)
$$

for any pair $\alpha_{1}, \alpha_{2} \in S_{R}(0)$ such that $\alpha_{1}^{n}=\alpha_{2}^{n}$.

But $P(x, y)$ is in $A\left(S_{R}(0)\right)$, and hence $P((x, y), z)=p_{(x, y)}\left(z^{n}\right)$ for some $p_{(x, y)}(z)$ in $A\left(S_{R}(0)\right)$. But, since $P=\varphi\left(z^{m} \cdot \chi_{\tau}\right), P$ is in $z^{m} \cdot A_{2}$ and there exists $H \in A_{2}$ such that $P=z^{m} \cdot H$.

Hence for $(x, y)$ in $G$ and $z$ in $S_{R}(0)$ we obtain

$$
z^{m} \cdot H((x, y), z)=p_{(x, y)}\left(z^{n}\right) .
$$

From (5.4) it follows that $z^{m} \cdot H((x, y), z)=z^{n} \cdot s_{(x, y)}\left(z^{n}\right)$ for some $s_{(x, y)}(z)$ in $A\left(S_{R}(0)\right)$. Then we obtain $H((x, y), z)=z^{n-m} \cdot s_{x, y}\left(z^{n}\right)$ for every $(x, y) \in G$ 
and $z \in S_{R}(0)$ for any $R<1$, and then by continuity for any $z \in \overline{\mathbb{D}}$. Then, clearly, if $n>m, H$ is in $z \cdot A_{2}$ and hence $\varphi\left(z^{m} \cdot \chi_{\tau}\right)=z^{m} \cdot H$ is in $z^{m+1} \cdot A_{2}$. But, as we showed above, $\varphi^{-1}\left(z^{m+1} \cdot A_{2}\right)=z^{m+1} \cdot A_{1}$ and $z^{m} \cdot \chi_{\tau} \notin z^{m+1} \cdot A_{1}$. Hence we have a contradiction, and thus $n \leq m$.

We treat the case $n<m$ in the same way, using $\varphi^{-1}$ instead of $\varphi$ and $A_{2}$ instead of $A_{1}$. Thus we obtain $n=m$.

\section{REFERENCES}

[AS] J. Arazy and B. Solel, Isometries of non-self-adjoint operator algebras. J. Funct. Anal. 90 (1990), 284-305. MR 91c:47085

[DP] L.M. De Alba and J. Peters, Classification of semi-crossed products of finite-dimensionals C*-algebras, Proc. Amer. Math. Soc. 95 (1985), 557-564. MR 87e:46088

$[\mathrm{H}] \quad$ K. Hoffman, Banach Spaces of Analytic Functions, Prentice-Hall, Englewood Cliffs, N.J., 1962. MR 24:A2844

[K] A.M. Kumjian, On $C^{*}$-diagonals, Can. J. Math. 38 (1986), 969-1008. MR 88a:46060

[MM] M.J. McAsey and P.S. Muhly, Representations of non-self-adjoint crossed products, Proc. Lond. Math. Soc. 47 (1983), 128-144. MR 85a:46039

[MS] P.S. Muhly and B. Solel, Subalgebras of groupoid $C^{*}$-algebras, J. Reine Angew. Math. 402 (1989), 41-75. MR 90m:46098

[P1] J. Peters, Semi-crossed products of $C^{*}$-algebras, J. Funct. Anal. 59 (1984), 498-534. MR 86e: 46063

[P2] J. Peters, On inductive limits of matrix algebras of holomorphic functions, Trans. Amer. Math. Soc. 299 (1987), 303-318. MR 88f:46130

[Ped] G.K. Pedersen, $C^{*}$-algebras and their automorphism groups, Academic Press, 1989. MR 81e: 46037

[R] J. Renault. A groupoid approach to $C^{*}$-algebras, Lecture Notes in Math., vol. 793, SpringerVerlag, 1980. MR 82h:46075

[Rick] C.E. Rickart, General theory of Banach algebras, Van Nostrand, 1960. MR 22:5903

[S] B. Solel, Applications of the asymptotic range to analytic subalgebras of groupoid $C^{*}$ algebras, J. Ergodic Theory Dynamical Systems 12 (1992), 341-358. MR 93m:46084

Department of Mathematics, Technion - Israel Institute of Technology, 3200 Haifa, ISRAEL

E-mail address: meshpigel@math.uwaterloo.ca 